\title{
Acoustic regularities in infant-directed vocalizations across cultures
}

Cody J. Moser*1, Harry Lee-Rubin ${ }^{1}$, Constance M. Bainbridge ${ }^{1}$, S. Atwood ${ }^{1,2}$, Jan Simson ${ }^{1}$, Dean Knox ${ }^{3}$, Luke Glowacki $^{4}$, Andrzej Galbarczyk ${ }^{5}$, Grazyna Jasienska ${ }^{5}$, Cody T. Ross ${ }^{6}$, Mary Beth Neff ${ }^{7}$, Alia Martin ${ }^{7}$, Laura K. Cirelli ${ }^{8,9}$, Sandra E. Trehub ${ }^{9}$, Jinqi Song ${ }^{10}$, Minju Kim ${ }^{11}$, Adena Schachner ${ }^{11}$, Tom A. Vardy ${ }^{12}$, Quentin D. Atkinson ${ }^{12,13}$, Jan Antfolk ${ }^{14}$, Purnima Madhivanan ${ }^{15,16,17,18}$, Anand Siddaiah ${ }^{19,20}$, Caitlyn D. Placek ${ }^{21}$, Gul Deniz Salali $^{22}$, Sarai Keestra ${ }^{22}$, Manvir Singh ${ }^{1,23}$, Scott A. Collins ${ }^{24}$, John Q. Patton ${ }^{25}$, Camila Scaff ${ }^{26}$, Jonathan Stieglitz $^{27,28}$, Cristina Moya ${ }^{29}$, Rohan R. Sagar ${ }^{30}$, Brian M. Wood ${ }^{31}$, Max M. Krasnow ${ }^{1}$, and Samuel A. Mehr*1,7,32

${ }^{1}$ Department of Psychology, Harvard University, Cambridge, MA 02138, USA

${ }^{2}$ Department of Psychology, University of Washington, Seattle, WA 98105, USA

${ }^{3}$ Department of Politics, Princeton University, Princeton, NJ 08544, USA

${ }^{4}$ Department of Anthropology, Pennsylvania State University, University Park, PA 16802, USA

${ }^{5}$ Department of Environmental Health, Faculty of Health Sciences, Jagiellonian University Medical College, 31-531 Krakow, Poland

${ }^{6}$ Department of Human Behavior, Ecology and Culture, Max Planck Institute for Evolutionary Anthropology, 04103 Leipzig, Germany

${ }^{7}$ School of Psychology, Victoria University of Wellington, Wellington 6012, New Zealand

${ }^{8}$ Department of Psychology, University of Toronto Scarborough, Toronto, Ontario M1C 1A4, Canada

${ }^{9}$ Department of Psychology, University of Toronto Mississauga, Mississauga, Ontario L5L 1C6, Canada

${ }^{10}$ Department of Mathematics, Univesity of California Los Angeles, Los Angeles, CA 90095, USA

${ }^{11}$ Department of Psychology, University of California, San Diego, La Jolla, CA 92093-0109, USA

${ }^{12}$ School of Psychology, University of Auckland, Auckland 1010, New Zealand

${ }^{13}$ Department of Linguistic and Cultural Evolution, Max Planck Institute for the Science of Human History, D-07745 Jena, Germany

${ }^{14}$ Department of Psychology, Åbo Akademi, 20500 Turku, Finland

${ }^{15}$ Department of Health Promotion Sciences, Mel \& Enid Zuckerman College of Public Health, University of Arizona, Tucson, AZ 85724 , USA

${ }^{16}$ Division of Infectious Diseases, College of Medicine, University of Arizona, Tucson, AZ 85724, USA

${ }^{17}$ Department of Family \& Community Medicine, College of Medicine, University of Arizona, Tucson, AZ 85724, USA

${ }^{18}$ Public Health Research Institute of India, Yadavgiri, Mysore 560020, India

${ }^{19}$ Department Of Epidemiology, Stempel School Of Public Health, Florida International University, Miami, FL 33157, USA

${ }^{20}$ Public Health Research Institute of India, Mysuru 570020, India

${ }^{21}$ Department of Anthropology, Ball State University, Muncie, IN 47306, USA

${ }^{22}$ Department of Anthropology, University College London, WC1H 0BW London, UK

${ }^{23}$ Department of Human Evolutionary Biology, Harvard University, Cambridge, MA 02138, USA

${ }^{24}$ School of Human Evolution and Social Change, Arizona State University, Tempe, AZ 85281, USA

${ }^{25}$ Division of Anthropology, California State University, Fullerton, CA 92831, USA

${ }^{26}$ Institute of Evolutionary Medicine, University of Zurich, 8006 Zürich, Switzerland

${ }^{27}$ Université Toulouse 1 Capitole, 31080 Toulouse Cedex 6, France

${ }^{28}$ Institute for Advanced Study in Toulouse, 31080 Toulouse Cedex 6, France

${ }^{29}$ Department of Anthropology, University of California, Davis, Davis, CA 95616, USA

${ }^{30}$ Future Generations University, Circle Ville, WV 26807, USA

${ }^{31}$ Department of Anthropology, University of California, Los Angeles, Los Angeles, CA 90095, USA

${ }^{32}$ Data Science Initiative, Harvard University, Cambridge, MA 02138, USA

*Corresponding author. Emails: cmoser@g.harvard.edu (C.J.M.); sam@wjh.harvard.edu (S.A.M.)

\begin{abstract}
Humans often produce vocalizations for infants that differ from vocalizations for adults. Is this property common across societies? The forms of infant-directed vocalizations may be shaped by their function in parent-infant communication. If so, infant-directed song and speech should be differentiable from adult-directed song and speech on the basis of their acoustic features, and this property should be relatively invariant across cultures. To test this hypothesis, we built a corpus of 1,614 recordings of infant- and adult-directed singing and speech produced by 411 people living in 21 urban, rural, and small-scale societies. We studied the corpus in a massive online experiment and in a series of acoustic analyses. Naïve listeners $(N=13,218)$ reliably identified infant-directed vocalizations as infant-directed, and adult-directed speech (but not songs) as adult-directed, at rates far higher than chance. Ratings of infant-directed song were the most accurate and the most consistent across all societies; infant-directed speech was accurately identified on average, but inconsistently across societies. To determine the mechanisms underlying these results, we extracted many acoustic features from each recording and identified those that most reliably characterize infant-directed song and speech across cultures, via preregistered exploratory-confirmatory analyses and machine classification. The features distinguishing infant- and adult-directed song and speech concerned pitch, rhythmic, phonetic, and timbral attributes; a hypothesis-free classifier with cross-validation across societies reliably identified all vocalization types, with highest accuracy for infant-directed song. Last, we isolated 12 acoustic features that were predictive of perceived infant-directedness; of these, two pitch attributes (median $\mathrm{F}_{0}$ and its variability) were by far the most explanatory. These findings demonstrate cross-cultural regularities in infant-directed vocalizations that are suggestive of universality; moreover, infant-directed song appears to be more cross-culturally stereotyped than infant-directed speech, informing hypotheses of the functions and evolution of both.
\end{abstract}

Keywords: vocalization, human infants, human parents, music, speech, form and function, cross-cultural 


\section{Background}

The forms of many animal signals are shaped by their functions, a link arising from production- and receptionrelated rules that help to maintain reliable signal detection within and across species ${ }^{1-6}$. This is especially true of vocal signals, where form-function links have been demonstrated across many species, including nonhuman primates ${ }^{3}$, meerkats $^{7}$, grackles ${ }^{8}$, frogs $^{9}$, and fish ${ }^{10}$.

The link between form and function in vocalizations is also evident from listeners' behavior. For example, humans ${ }^{11}$, red deer ${ }^{12}$, and canines ${ }^{13}$ reliably detect the intentions of heterospecific signalers on the basis of the sounds of their signals. A classic demonstration of this fact is the ability of some species to eavesdrop on the alarm signals of other species, whether or not their own species has an extended vocal repertoire ${ }^{14,15}$.

In humans, an area of particular importance for effectively transmitting vocal signals is between parents and infants. This is because human infants are especially helpless to manage their own nutrition, safety, and comfort. Infants use a distinctive alarm signal to elicit care from those around them - they cry ${ }^{16}$. In response, adults and children produce infant-directed vocalizations, which are known to differ reliably from adult-directed vocalizations in at least some societies, in the form of speech ${ }^{17,18}$ or song ${ }^{19-21}$.

Are the forms and functions of infant-directed vocalizations linked, like the vocal signals of many other species? Fernald ${ }^{22}$ noted that a number of features of infant-directed vocalizations observed in Western societies follow Wiley's criteria for signal detection in biological systems ${ }^{5}$. Many others have proposed ways in which infant-directed and adult-directed speech might differ; for example, when compared to adultdirected speech, infant-directed speech may have longer voice-onset times ${ }^{23}$; higher $\mathrm{pitch}^{24,25}$; more formant variability $^{26}$; longer and more carefully articulated vowels ${ }^{27,28}$, with an upwards-shifted vowel space ${ }^{29}$; more repetition, with longer pitch curves ${ }^{30}$; and more temporal amplitude variability ${ }^{31}$. Many of these features are predicted by functional accounts of stereotyped infant-directed speech, which propose that it facilitates word segmentation ${ }^{32}$, distinction of sound categories ${ }^{33}$, the elicitation of infant attention ${ }^{34}$, or parent-infant communication at a distance ${ }^{35}$.

Infants appear to be receptive to at least some of these features, across at least some languages. For example, the ManyBabies Consortium study of 2,329 monolingual infants found reliable preferences for North American English infant-directed speech (relative to North American English adult-directed speech), even when, for more than half of the infants, North American English was not their native language ${ }^{36}$. Infants also have expectations about the infant-directed speech they hear: they look longer at videos of infant-directed speech being directed to an adult-like character, relative to videos of infant-directed speech being directed to an infant-like character, across several languages ${ }^{37}$.

Whether or not infant-directedness is characterized by universal acoustic features is unknown, however. Infant-directed speech has rarely been studied outside of Western, Educated, Industrialized, Rich, or Democratic (WEIRD) societies $^{38}$, despite a longstanding interest in cross-cultural regularities in infant development ${ }^{39,40}$. No corpora have systematically measured the acoustics of infant-directed speech across a variety of societies, and the pattern of results in smaller studies is unclear.

The prosody of infant-directed speech is similar across tonal and non-tonal languages ${ }^{41,42}$; across French, Italian, German, Japanese, and British and American English ${ }^{43}$; and across Fijian, Kenyan, and North American adults ${ }^{44}$. Across North American English, Swedish, and Russian, infant-directed speech includes vowel accentuation to a more extreme extent than does adult-directed $\operatorname{speech}^{28}$. Adults from the Shuar, a South American hunter-horticulturalist group, accurately distinguish infant- from adult-directed speech in recordings of North American English mothers ${ }^{17}$; they do so, in part, on the basis of pitch. This finding echoes reports of raised pitch in Lebanese infant-directed speech ${ }^{45}$. In contrast, the infant-directed speech of fathers in a small-scale Vanuatuan society is rather different in pitch and speech rate than that of North American fathers ${ }^{46}$. And the timbre of infant-directed speech differs from adult-directed speech in ten languages, though with very small samples of speakers ${ }^{18}$. (Note that several studies of the frequency of occurrence of infant-directed speech have been conducted in non-WEIRD and small-scale societies ${ }^{47,48}$, but these address a separate question from what acoustic features characterize infant-directed vocalizations when they do occur). 
In the domain of music, Mehr and Krasnow proposed that infant-directed song emerged through arms-race coevolution as an honest signal of parental attention, with acoustic forms elaborated from other vocalizations, such as non-human primate contact calls, so as to provide infants with reliable information that they were being kept safe ${ }^{49}$. This idea is supported by at least three forms of evidence. First, infant-directed song modulates infant arousal, whether the songs are familiar ${ }^{50}$ or not ${ }^{51}$, and delays the onset of infant distress longer than does infant-directed speech ${ }^{52}$. Second, people with genomic imprinting disorders, which are characterized by altered parental investment behaviors, such as those related to food consumption ${ }^{53,54}$, also have altered music perception ability and responses to music ${ }^{55,56}$. Last, consistent with classic ideas in the psychology of music ${ }^{57-59}$ substantial evidence demonstrates that lullabies, one typical form of infantdirected song, are a human universal: singing is associated with infant care across the ethnographies of a representative sample of human small-scale societies, even after correcting for reporting biases ${ }^{21}$, and parents use singing to calm infants in several of the most genetically distant human societies, the Hadza, Mbuti, and !Kung San hunter-gatherers of East, Central, and South Africa ${ }^{60-62}$. Other forms of infant-directed song, like excitatory play songs and singing games for children, also appear to be widespread ${ }^{21,63}$, and parents produce them often ${ }^{64}$.

The universality of infant-directed song is also supported by evidence showing that its acoustics differ from those of other forms of music. For example, naïve listeners reliably identify lullabies as infant-directed in a cross-culturally representative sample of vocal music, both when rating multiple functions (e.g., rating the songs more highly as "used to soothe a baby" than "used for dancing" ${ }^{20}$ ) and in a forced-choice classification $\operatorname{task}^{21}$. This finding echoes earlier work, wherein adult listeners were able to distinguish lullabies from love songs recorded in some foreign societies ${ }^{19}$. And machine classifiers reliably distinguish lullabies from healing, dance, and love songs based only on pitches and rhythms of the vocalizations, as opposed to acoustic features merely associated with the vocalization, such as the sound of an infant crying ${ }^{21}$.

In sum, while infant-directed song and speech seem to appear universally, the ways in which they are acoustically distinct from other vocalizations are not fully understood, nor is it clear whether those acoustic distinctions are themselves universal. This makes it difficult to evaluate the theories of the functions of infantdirected vocalizations mentioned above $\mathrm{e}^{32-35,49,57-59}$, all of which imply the presence of universal acoustic structure in infant-directed speech or song.

To explore these questions, we built a corpus of infant-directed song, infant-directed speech, adult-directed song, and adult-directed speech from a diverse set of 21 human societies. Each participant provided all four recordings, enabling within-person analyses of the differences between the vocalization types. The corpus is open-access at https://osf.io/m5yn2. Here, we report tests of the cross-cultural regularity of the acoustics of infant-directed song and speech, studied via (1) a large-scale listener experiment, where naïve adults recruited online from many countries were asked to discriminate between infant-directed and adult-directed vocalizations in the corpus; and (2) a series of acoustic analyses, to determine reliably-occurring differences in the production and perception of infant-directed vocalizations worldwide.

\section{Vocalization corpus}

We built a corpus of recordings of infant-directed song, infant-directed speech, adult-directed song, and adult-directed speech. Participants $(N=411)$ living in 21 societies (Figure 1 and Table 1$)$ produced each of these vocalizations, respectively, with a median of 15 participants per society (range: 6-57). From those participants for whom information was available, most were female (86\%) and nearly all were parents and/or grandparents (95\%). Recordings were collected by principal investigators and/or staff at their field sites, all using the same data collection protocol. They translated instructions to the native language of the participants, following the standard research practices at each site.

For infant-directed song and infant-directed speech, participants sang or spoke to their infant as if they were fussy, where "fussy" could refer to anything from frowning or mild whimpering to a full tantrum (note that each language had its own word for "fussy", suggesting that participants had an intuitive understanding of it). For most participants (90\%) an infant was physically present during the recording (the infants were $48 \%$ female; mean age $11.4 \mathrm{mo}$; $\mathrm{SD}=0.6 \mathrm{mo}$; range: $0.5-48)$. When an infant was not present, participants were 


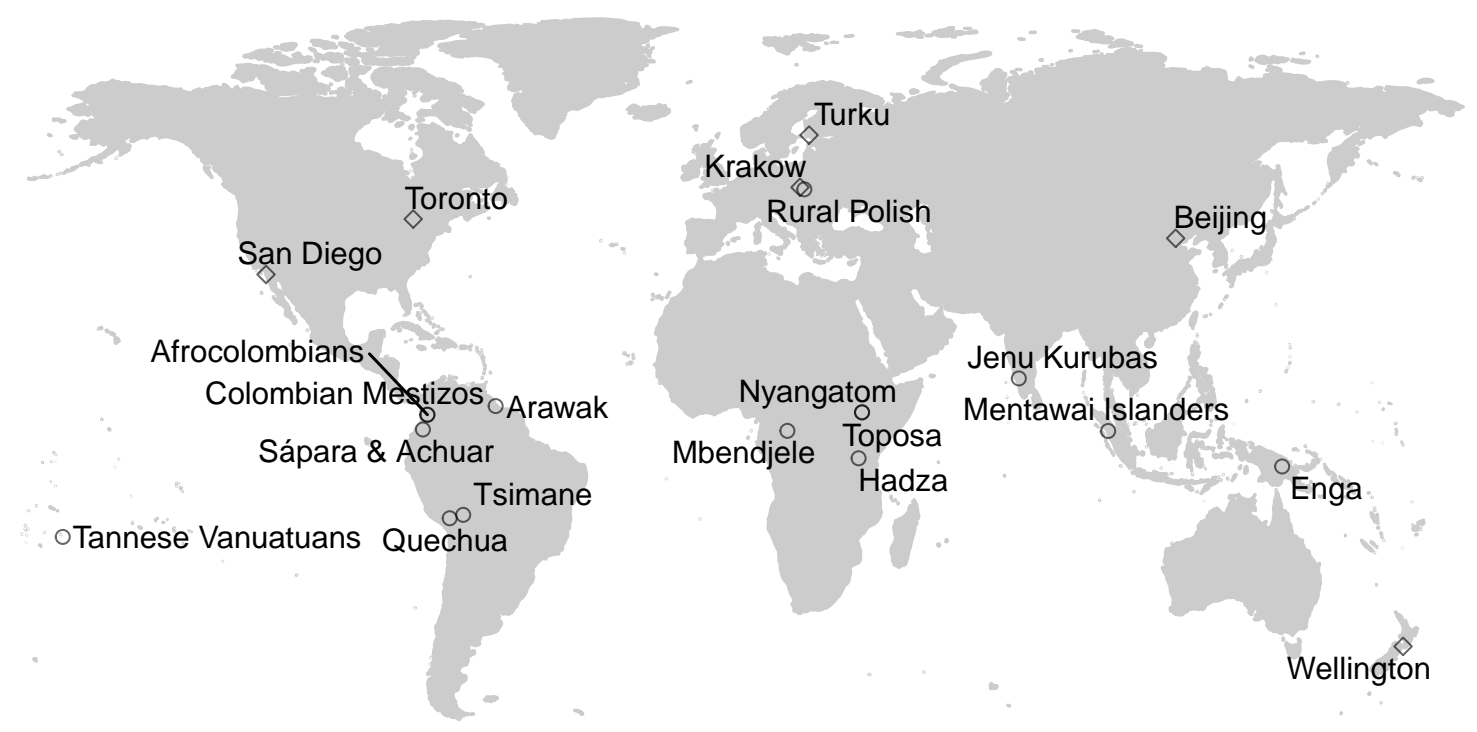

Figure 1. Societies from which vocalizations were recorded. Diamonds denote urban societies; circles denote rural or small-scale societies.

asked to imagine that they were vocalizing to their own infant or grandchild, and simulated their infantdirected vocalizations. For adult-directed song, participants sang a song that was not intended for infants; they also stated what that song was for (e.g., "a celebration song"). For adult-directed speech, participants spoke to the researcher about a topic of their choice (e.g., they described their daily routine).

In all cases, participants were free to determine the content of their vocalizations. This was intentional: imposing a specific content category on their vocalizations (e.g., "sing a lullaby") would likely alter the acoustic features of their vocalizations, which are known to be influenced by experimental contexts ${ }^{65}$.

All recordings were made with Zoom H2n digital field recorders, using foam windscreens (where available). To ensure that participants were audible along with researchers (who stated information about the participant and environment before and after the vocalizations), recordings were made with a 360 -degree dual-X/Y microphone pattern. This produced two uncompressed stereo audio files (WAV) per participant at $44.1 \mathrm{kHz}$; we only analyzed audio from the two-channel file on which the participant was loudest.

We manually extracted the longest continuous and uninterrupted section of audio from each of the four samples per participant (i.e., isolating vocalizations by the participant from interruptions from other speakers, the infant, and so on), using Adobe Audition. We then used the silence detection tool in Praat ${ }^{66}$, with minimum sounding intervals at 0.1 seconds and minimum silent intervals at 0.3 seconds, to remove all portions of the audio where the participant was not speaking (i.e., the silence between vocalization phrases). These were manually concatenated in Python, producing denoised recordings, which were subsequently checked manually to ensure minimal loss of content. Further details of the acoustic analyses are in the Supplementary Information.

\section{$3 \quad$ Naïve listener experiment}

We used the citizen science platform https://themusiclab.org to play excerpts of each item in the corpus to listeners who were unaware of the type of vocalization they heard and who were presumably unfamiliar with many of the societies in which the vocalizations were recorded. This experiment is similar in style to other studies of form and function in vocalization ${ }^{11,19-21}$. 


\begin{tabular}{|c|c|c|c|c|c|c|}
\hline Region & Sub-Region & Society & Language & Language Family & Subsistence Type & $N$ \\
\hline \multirow[t]{4}{*}{ Africa } & Central Africa & $\begin{array}{l}\text { Mbendjele } \\
\text { BaYaka }\end{array}$ & Mbendjele & Niger-Congo & Hunter-Gatherer & 60 \\
\hline & Eastern Africa & Hadza & Hadza & Hadza & Hunter-Gatherer & 38 \\
\hline & & Nyangatom & Nyangatom & Nilotic & Pastoralist & 56 \\
\hline & & Toposa & Toposa & Nilotic & Pastoralist & 60 \\
\hline \multirow[t]{3}{*}{ Asia } & East Asia & Beijing & Mandarin & Sino-Tibetan & Urban & 124 \\
\hline & South Asia & Jenu Kurubas & Kannada & Dravidian & Other & 80 \\
\hline & Southeast Asia & $\begin{array}{l}\text { Mentawai } \\
\text { Islanders }\end{array}$ & Mentawai & Austronesian & Horticulturalist & 60 \\
\hline \multirow[t]{3}{*}{ Europe } & Eastern Europe & Krakow & Polish & Indo-European & Urban & 44 \\
\hline & & Rural Poland & Polish & Indo-European & $\begin{array}{l}\text { Intensive } \\
\text { Agriculturalists }\end{array}$ & 55 \\
\hline & Scandinavia & Turku & $\begin{array}{l}\text { Finnish \& } \\
\text { Swedish }\end{array}$ & $\begin{array}{l}\text { Uralic and } \\
\text { Indo-European }\end{array}$ & Urban & 80 \\
\hline \multirow[t]{2}{*}{$\begin{array}{l}\text { North } \\
\text { America }\end{array}$} & North America & San Diego & $\begin{array}{l}\text { English } \\
\text { (USA) }\end{array}$ & Indo-European & Urban & 116 \\
\hline & & Toronto & $\begin{array}{l}\text { English } \\
\text { (Canadian) }\end{array}$ & Indo-European & Urban & 198 \\
\hline \multirow[t]{3}{*}{ Oceania } & Melanesia & $\begin{array}{l}\text { Tannese } \\
\text { Vanuatuans }\end{array}$ & Bislama & $\begin{array}{l}\text { Indo-European } \\
\text { Creole }\end{array}$ & Horticulturalist & 90 \\
\hline & & Enga & Enga & $\begin{array}{l}\text { Trans-New } \\
\text { Guinea }\end{array}$ & Horticulturalist & 22 \\
\hline & Polynesia & Wellington & $\begin{array}{l}\text { English (New } \\
\text { Zealand) }\end{array}$ & Indo-European & Urban & 228 \\
\hline \multirow[t]{6}{*}{$\begin{array}{l}\text { South } \\
\text { America }\end{array}$} & Amazonia & Arawak & $\begin{array}{l}\text { English } \\
\text { Creole }\end{array}$ & Indo-European & Other & 48 \\
\hline & & Tsimane & Tsimane & Moseten-Tsimane & Horticulturalist & 51 \\
\hline & & $\begin{array}{l}\text { Sápara \& } \\
\text { Achuar }\end{array}$ & $\begin{array}{l}\text { Quechua \& } \\
\text { Achuar }\end{array}$ & $\begin{array}{l}\text { Quechuan \& } \\
\text { Jivaroan }\end{array}$ & Horticulturalist & 59 \\
\hline & Central Andes & Quechua & Quechua & Quechuan & Agro-Pastoralist & 49 \\
\hline & $\begin{array}{l}\text { Northwestern } \\
\text { South America }\end{array}$ & Afrocolombians & Spanish & Indo-European & Horticulturalist & 53 \\
\hline & & $\begin{array}{l}\text { Colombian } \\
\text { Mestizos }\end{array}$ & Spanish & Indo-European & $\begin{array}{l}\text { Commercial } \\
\text { Economy }\end{array}$ & 43 \\
\hline
\end{tabular}

Table 1. Societies from which recordings were gathered. $N$ refers to the total number of recordings from each site, not the number of participants.

\subsection{Methods}

We analyzed all data available at the time of writing this manuscript from the "Who's Listening?" game at https://themusiclab.org/quizzes/ids, a jsPsych ${ }^{67}$ experiment distributed via Pushkin ${ }^{68}$ to both desktop and mobile web browsers. Participants $(N=13,218$; gender: 4,405 female, 7,043 male, 176 other, 1,594 did not disclose; age: median 31 years, interquartile range 23-43) listened to at least 1 and at most 16 vocalizations drawn at random from the corpus, for a total of 164,759 ratings (infant-directed song: $n=47,798$; infantdirected speech: $n=38,913$; adult-directed song: $n=41,277$; adult-directed speech: $n=37,071$ ). This yielded over 100 ratings per vocalization (median $=117$; interquartile range 107-154) and thousands of ratings for each society (median $=6,394$; interquartile range: $4,664-9,569)$. Most participants $(n=7,241)$ 
played the full game, listening to all 16 songs. Participants self-reported living in 109 different countries and speaking 96 different native languages; roughly half the participants were native English speakers from the United States. We excluded excerpts less than 10 seconds in duration from the online experiment, studying 1405 excerpts in total (with representation from all societies).

Participants were asked to classify each vocalization as either infant- or adult-directed (Figure S1), as quickly as possible, either by pressing a key corresponding to a drawing of an infant or adult face (when the participant used a desktop computer) or by tapping one of the faces (when the participant used a tablet or smartphone). As soon as they made a choice, playback stopped. They were given corrective feedback along with a score at the end of the experiment. Because each instance of the experiment included a new random draw of recordings, we did not exclude participants who disclosed that they had played it more than once $(n=279)$; note, however, that given a random draw of 16 vocalizations from the truncated corpus of 1405 in each instance of the experiment, repeat plays for the 279 participants who played more than once are expected to be rare.

We analyzed the patterns of successful identification of vocalization target across the full corpus and within each society, using both the raw identification accuracy and $d$-prime scores. We also analyzed response time from the onset of each recording, for the subset of responses that were accurate, to explore the speed with which participants made accurate inferences about vocalization types.

\subsection{Results}

We computed an average score for each vocalization, by averaging across all listeners, and used them as the raw data for the following analyses. Corpus-wide, scores were above chance level, at $65.3 \%$ correct (SD $=14.8 \%, 95 \%$ CI: $[63.9 \%, 66.8 \%] ; t=20.9, p<.0001$, one-sample $t$-test relative to $50.0 \%)$. Accuracy varied substantially, however, as a function of the vocalization type (Figure 2A): infant-directed song was identified most accurately $(79.7 \%$ correct), followed by adult-directed speech $(75.4 \%)$, and infant-directed speech $(68.0 \%)$; all these were well above chance $(p$ s $<.0001)$. In contrast, adult-directed song was reliably classified incorrectly, with only $38.4 \%$ accuracy (below chance at $p<.0001$ ). Here there was also substantial consistency across societies, with all but 2 showing an identical ordering of identification accuracy (in these remaining 2 societies, Wellington and San Diego, infant-directed speech was the highest-accuracy vocalization type).

To examine the degree to which these results held worldwide, we collapsed scores for the vocalizations from each society, in isolation, and analyzed each vocalization type independently (Figure S2; n.b., this analysis substantially reduces the sample size, as some societies had very few recordings available in the naïve listener experiment).

For infant-directed song, the result replicated robustly across societies: infant-directed songs were identified as infant-directed at a significantly higher rate than chance in 19 of 21 sites. In the remaining two societies, perceived infant-directedness trended above chance (Papua New Guinea: $M=.603$; Quechua: $M=.689$ ) but these sites had only 6 and 5 infant-directed songs, respectively, making it difficult to interpret their non-significant test statistics. Similarly, adult-directed speech was reliably identified as adult-directed in 19 of 21 sites, with trending results in the remaining two sites (Arawak: $M=.552, N=2$; Sápara/Achuar: $M$ $=.605, N=13)$.

These results contrast, however, with the identification of infant-directed speech: here, accuracy replicated in only 9 societies, fewer than half of those represented in the corpus. The societies where the naïve listeners failed to identify infant-directed speech accurately tended to be small-scale, including the Hadza, Tsimane, Mbendjele, Toposa, Nyangatom, and Mentawai Islanders (see Figure S2).

To ensure that the above findings were not attributable to response biases, we repeated the overall result using a $d$-prime analysis, which measures accuracy after adjusting for the base rates of response, which were skewed somewhat toward infant-directedness (approximately $60 \%$ of items were classified as infantdirected, despite only half actually being infant-directed). This analysis confirmed the main finding reported above (infant-directed song: $d^{\prime}=1.11$; adult-directed speech: $d^{\prime}=1.30$; infant-directed speech: $d^{\prime}=0.93$; 
(A)

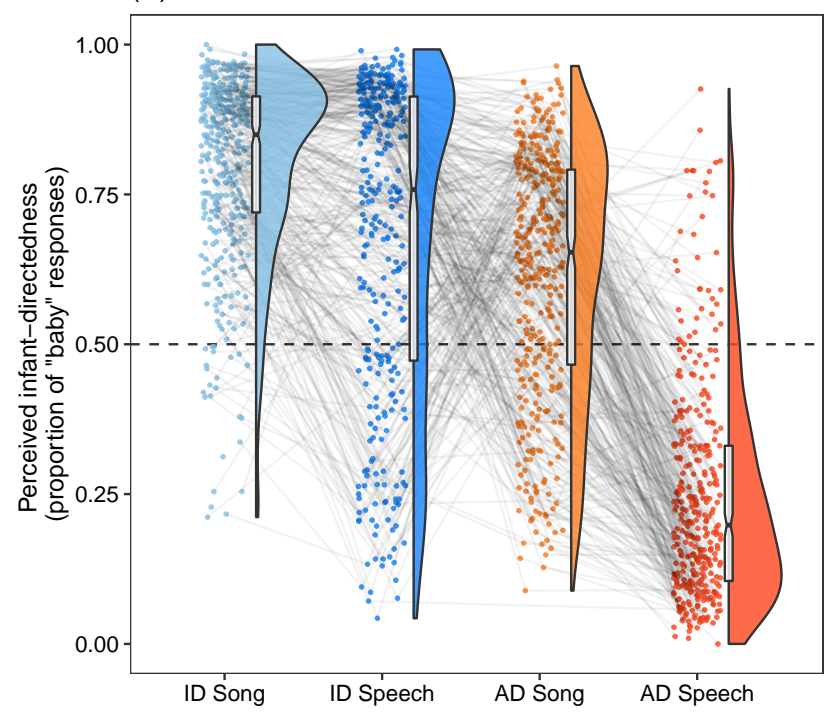

(B)

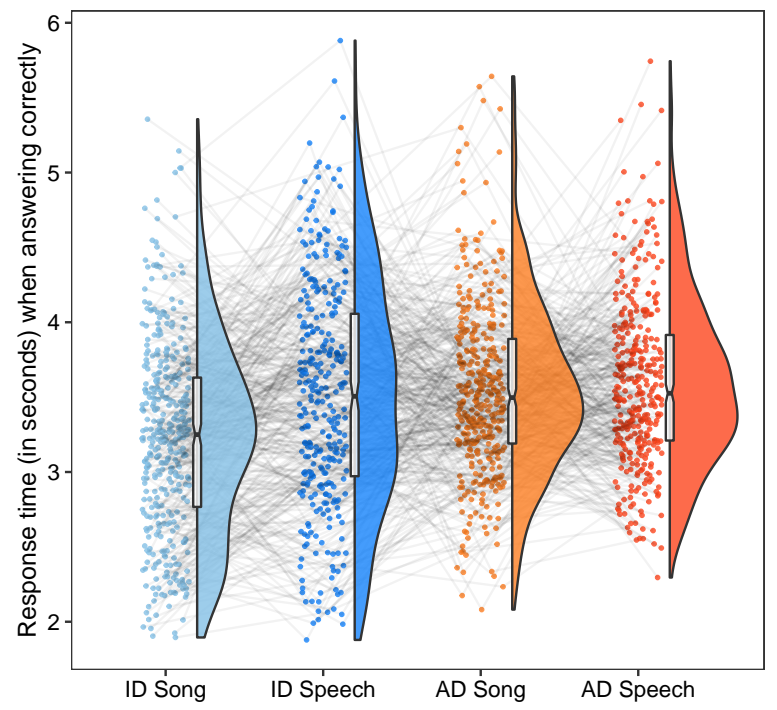

Figure 2. Results of the naïve listener experiment. (A) Listeners accurately identify infant-directed song and infant-directed speech as directed towards infants, and adult-directed speech as directed towards adults; however, they do not identify adult-directed song as directed toward adults. The horizontal dotted line represents chance level of 0.50 . (B) When responding correctly, listeners are fastest to identify infantdirected song, followed by infant-directed speech, adult-directed song, and adult-directed speech. In both panels, the points indicate averages for each recording; the gray lines connecting the points indicate the groups of vocalizations produced by the same participant; the half-violins are kernel density estimations; and the boxplots represent the medians, interquartile ranges, and $95 \%$ confidence intervals (indicated by the notches). Abbreviations: infant-directed (ID); adult-directed (AD).

adult-directed song: $d^{\prime}=-0.07 ; d^{\prime}$ scores greater than 0 represent significant results after adjusting for false positives).

Given theoretically-derived predictions that specifically concern the function of infant-directed singing ${ }^{49}$, and following our preregistered analysis plan (at https://osf.io/5r72u) for acoustic feature comparisons across vocalization types, we tested for differences in perceived infant-directedness across three comparisons of the vocalizations: (1) infant-directed vs. adult-directed vocalizations, overall; (2) infant-directed song vs. adultdirected song; and (3) infant-directed song vs. infant-directed speech.

In all cases, we analyze within-voice differences in perceived infant-directedness (e.g., for all voices, comparing the proportion of "baby" responses for infant-directed songs to infant-directed speech produced by the same voice). This procedure ensures that participant-wise differences in voice characteristics cannot account for differences in the perceived infant-directedness of each vocalization.

We found substantial support for all three predictions (Figure 2A). Perceived infant-directedness was higher in infant-directed vocalizations (proportion of "baby" responses; $M=.743, S D=.187,95 \%$ CI [.724, .762]) than adult-directed vocalizations, overall $(M=.448, S D=.182,95 \%$ CI $[.430, .467] ; t(372)=20.8, p<$ $.0001, d=2.07$, paired $t$-test); higher in infant-directed song $(M=.799, S D=.152,95 \%$ CI $[.783, .815])$ than adult-directed song $(M=.615, S D=.208,95 \%$ CI $[.593, .637] ; t(348)=13.4, p<.0001, d=1.29)$; and higher in infant-directed song $(M=.806, S D=.152,95 \%$ CI $[.789, .824])$ than infant-directed speech $(M=.688, S D=.263,95 \%$ CI $[.659, .718] ; t(301)=8.92, p<.0001, d=0.83)$.

Response time analyses paralleled these findings (Figure 2B). When restricting the sample to correct responses, participants answered more quickly for infant-directed vocalizations (in seconds, $M=3.34, S D=$ $0.61,95 \%$ CI $[3.28,3.40])$ than adult-directed vocalizations $(M=3.58, S D=0.46,95 \%$ CI $[3.53,3.62]$; 
$t(372)=6.27, p<.0001, d=0.54$, paired $t$-test $)$; more quickly for infant-directed song $(M=3.24, S D=$ $0.65,95 \%$ CI $[3.17,3.31])$ than adult-directed song $(M=3.54, S D=0.59,95 \%$ CI $[3.47,3.60] ; t(348)=$ $6.99, p<.0001, d=0.70)$; and more quickly for infant-directed song $(M=3.19, S D=0.64,95 \%$ CI $[3.12$, $3.27])$ than infant-directed speech $(M=3.50, S D=0.76,95 \%$ CI $[3.41,3.58] ; t(301)=6.89, p<.0001, d=$ 0.70). Because web-based participants may halt their participation during a trial (producing extremely long response times) or answer quickly at random (producing extremely short response times), in these analyses we removed observations below the 1st and above the 99th percentiles. Also note that in these and the previous paragraph's analyses, summary statistics vary across the comparisons, because a small number of participants did not provide all four of the vocalization types, and because recordings with a duration of less than 10 seconds were excluded from the online experiment. Effect sizes $(d s)$ were computed using the overall standard deviation of accuracy, for consistency across tests.

\subsection{Interim discussion}

The naïve listener experiment provides evidence that infant-directed vocalizations from around the world are discriminable from adult-directed vocalizations. This effect was most consistent for infant-directed song, which was reliably identified within each society represented in the corpus; while infant-directed speech was reliably identified on average, its society-wise results were less consistent.

Why are listeners so good at identifying infant-directed song? Cross-cultural identification of infantdirectedness in music might be due to universal acoustic cues, as predicted from functional accounts of infant-directed vocalizations. In the rest of this paper, we analyze the acoustic features that most reliably characterize infant-directed song, using both confirmatory and hypothesis-free methods, and test the degree to which these features explain overall ratings in the naïve listener experiment.

\section{Analysis of acoustic features}

We studied a broad range of acoustic features in each vocalization, using Praat ${ }^{66}$, MIRtoolbox ${ }^{69}$, discrete Fourier transforms for rhythmic variability ${ }^{70}$, and normalized pairwise variability indices ${ }^{71}$. The acoustic features consisted of measurements of pitch (e.g., $F_{0}$, the fundamental frequency), timbre (e.g., roughness), and rhythm (e.g., tempo); all summarized over time. We extracted a variety of summary variables for each feature, producing 94 variables in total. For example, in the domain of pitch, we included 9 summaries of the feature $\mathrm{F}_{0}$ (mean, median, minimum, maximum, range, standard deviation, first quartile, third quartile, and interquartile range), and similar summaries for $\mathrm{F}_{1}$ and $\mathrm{F}_{2}$, change in $\mathrm{F}_{0}$, and so on. A codebook for all features is in Table S1.

We ran three sets of analyses. First, we randomly selected half the recordings in the corpus for exploratory analyses, confirming the results on the other half of the corpus, so as to reduce the risk of Type I error. Of particular interest in these analyses were the set of confirmatory hypotheses that we preregistered, following the exploratory analysis, based on functional theories of infant-directed vocalization ${ }^{32-35,49,57-59}$ and general principles of signal detection ${ }^{5}$.

Second, we used an hypothesis-free machine learning tool, least absolute shrinkage and selection operator (LASSO) classification ${ }^{72}$. To assess how distinct each vocalization type was, in terms of its acoustic features, we evaluated classification accuracy with a cross-validation procedure in which each society's recordings were classified using statistical models trained on the 20 other societies. This design allows us to gauge whether acoustic patterns are consistent cross-culturally (following prior research using a similar classification task ${ }^{21}$ ). The algorithm also includes a variable selection step to identify the specific acoustic features that most reliably characterize each vocalization type across the 21 societies.

Third, we explored the degree to which the convergent results of the first two analyses - namely, the acoustic features that most reliably characterized infant-directed song and infant-directed speech - can explain the results of the naïve listener experiment. We regressed an infant-directedness score for each recording on the acoustic features that predicted infant-directedness in both analyses, using a strict inclusion criterion 
and a conservative correction for multiple tests, to determine the core set of acoustic features characterizing infant-directedness worldwide.

\subsection{Exploratory-confirmatory analyses}

In exploratory analyses, we fitted a multi-level mixed-effects model for each acoustic feature, adjusting for subject and society and using three predictors: (1) target (infant-directed or adult-directed); (2) utterance type (song or speech); and (3) their interaction. For each model, we tested three linear combinations, to examine differences between (1) infant- and adult-directed vocalizations, overall; (2) infant-directed song and adult-directed song; and (3) infant-directed song and infant-directed speech. This procedure, which was preregistered, mirrors the pairwise comparison analyses in the naïve listener experiment. The linear combinations were evaluated with one-tailed $z$-tests, using an alpha level of .05. We did not correct for multiple tests in these analyses because the exploratory-confirmatory design restricts the number of tests to those with a strong directional prediction. We did all this with half the corpus, weighted by participant.

In the course of the exploratory analyses, we noted a small number of extreme outliers, typically attributable to anomalies in the recording environment (e.g., loud wind). As such, before running confirmatory analyses, we Winsorized all features at the lowest and highest 5 percentile ranks, and also restricted the set of features analyzed to those less sensitive to extreme observations (e.g., using the median as a measure of central tendency rather than the mean). These data were used for all subsequent analyses. This decision had no impact on the interpretation of results, but is preferable to trimming extreme values ${ }^{73}$; an alternate method, imputing extreme values with the mean observation for each feature, yielded comparable results.

We ran confirmatory models on the subset of acoustic features that were found to distinguish vocalization types in exploratory findings (Table S2), using the other half of the corpus. We were particularly interested in those features for which we had a preregistered directional prediction. These included predictions derived from Mehr and Krasnow ${ }^{49}$, suggesting that infant-directed song may universally have longer attack envelopes and pitch contours than infant-directed speech, as well as slower amplitude decay, lower $\mathrm{F}_{0}$, clearer signal-to-noise parameters, and greater vowel prolongation and stability; slower tempo ${ }^{22}$, differential rhythmic variance ${ }^{70,74}$, less roughness ${ }^{75}$, and shifted vowel spaces $^{29,76}$. The full list of theoretically-motivated hypotheses is at the preregistration (https://osf.io/5r72u) and is summarized in Table S3.

The exploratory-confirmatory procedure yielded 46 significant differences across the three comparison types, confirming some of the preregistered predictions, in terms of pitch, formant, timbre, and temporal features (Figure 3 and Table S4). For example, relative to adult-directed vocalizations, infant-directed vocalizations had a higher pitch and wider pitch variability, faster rates of pitch change and more variability in those rates, and a wider pitch space; a faster rate of vowel space change and more variability in that space; more intensity changes and more variability in intensity; a lower energy profile; and lower inharmonicity. We found similar differences in the other two comparison types, including a few additional acoustic features, such as the normalized pairwise variability index (nPVI, a measure of durational contrast) and attack slopes (a measure of the amplitude change in the onset of acoustic events). The full results are in Table S4.

\subsection{Hypothesis-free classification}

To validate the results of the exploratory-confirmatory models, we used a hypothesis-free LASSO-regularized categorical classifier ${ }^{72}$ to identify the four different vocalization types on the basis of their acoustic features alone. Cross-cultural accuracy was assessed using society-wise leave-one-out cross-validation, as in previous research $^{21}$. We then rotated the held-out society 20 more times, to analyze accuracy across all 21 societies. The classifier used acoustic features standardized within-voices, eliminating between-voice variability in the acoustic features.

The classifier accurately identified $70.5 \%$ of held-out recordings from unseen societies ([62.9\%, 78.0\%]; $95 \%$ CIs from corrected and resampled $t$-tests ${ }^{77}$ ), far above chance level of $25 \%$. This finding justifies a strong claim of corpus-wide consistency: to predict vocalization types in a given society, the classifier only used information available from other societies, and did so with a high degree of accuracy (Figure 4A). 
bioRxiv preprint doi: https://doi.org/10.1101/2020.04.09.032995; this version posted April 11, 2020. The copyright holder for this preprint (which was not certified by peer review) is the author/funder, who has granted bioRxiv a license to display the preprint in perpetuity. It is made available under aCC-BY-NC-ND 4.0 International license.

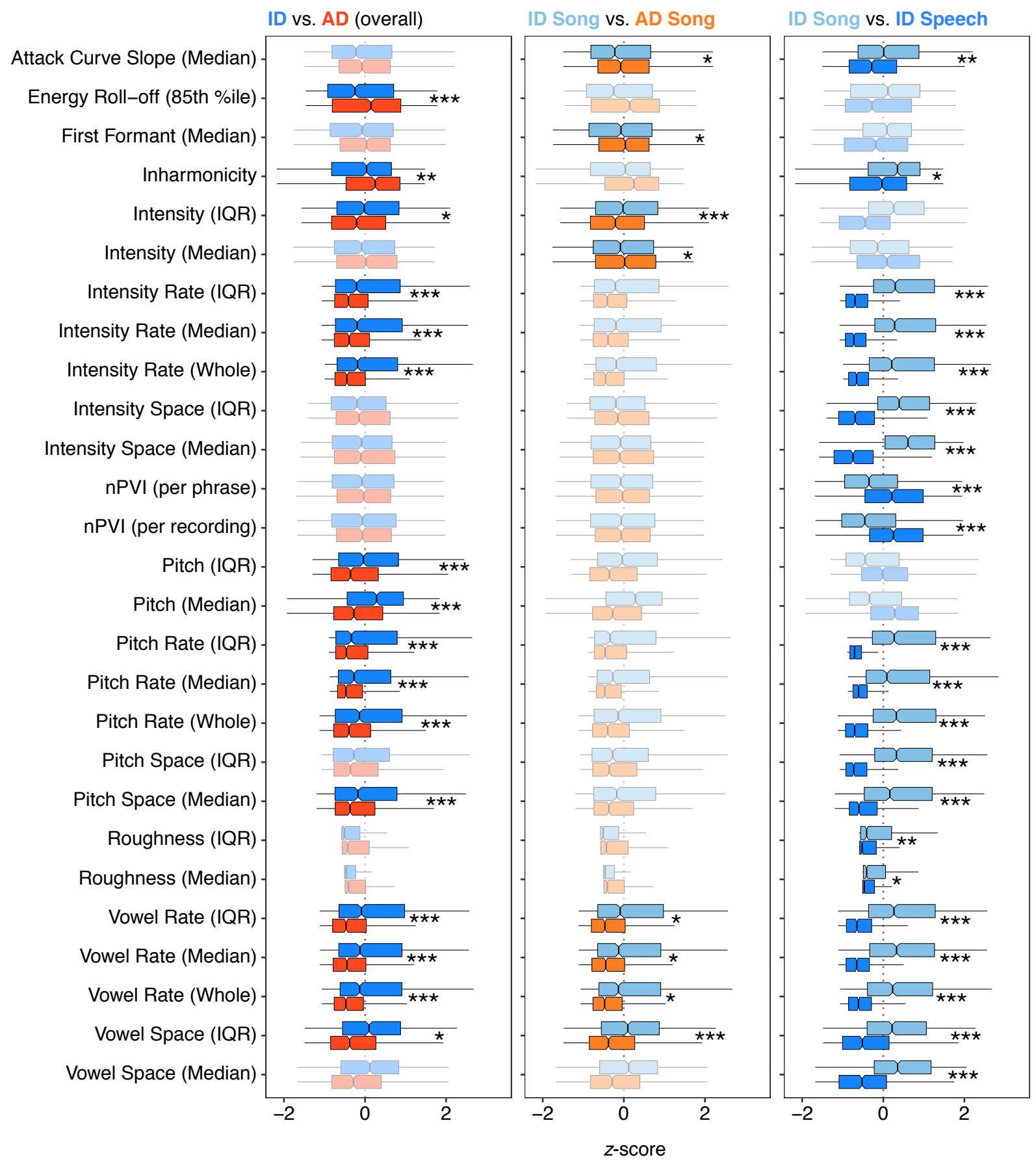

Figure 3. Confirmatory results. The boxplots represent the 25 acoustic features with a significant difference in at least one main comparison (e.g., infant-directed song vs. infant-directed speech, in the right panel), in both the exploratory and confirmatory analyses. All variables are normalized across participants. The boxplots represent the median and interquartile range; the whiskers indicate $1.5 \times$ IQR; and the notches represent the $95 \%$ confidence intervals of the medians. Faded comparisons did not reach significance in exploratory analyses. Abbreviations: infant-directed (ID); adult-directed (AD). Significance values are computed via linear combinations, following multi-level mixed-effects models. ${ }^{* * *} p<.001 ;{ }^{* *} p<.01 ;{ }^{*} p<$ .05 
(A)

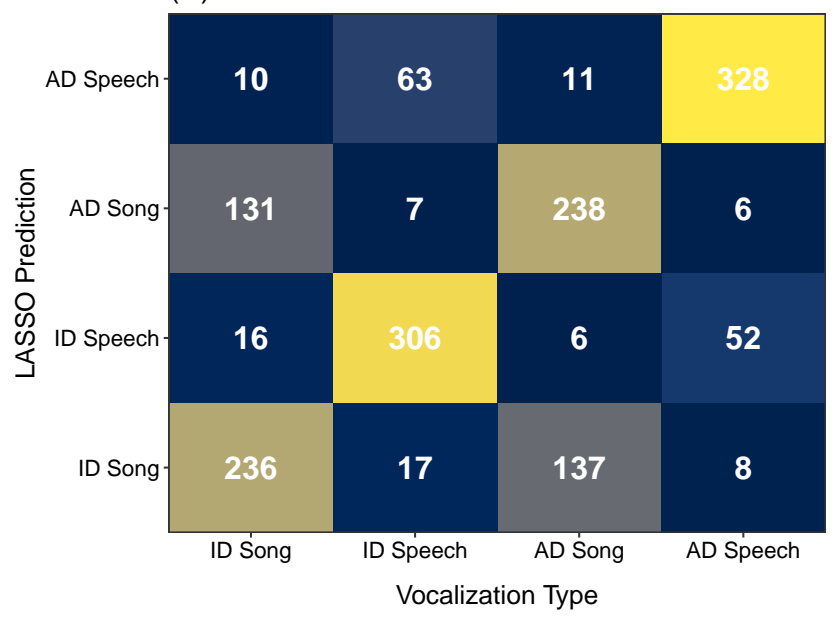

(B)

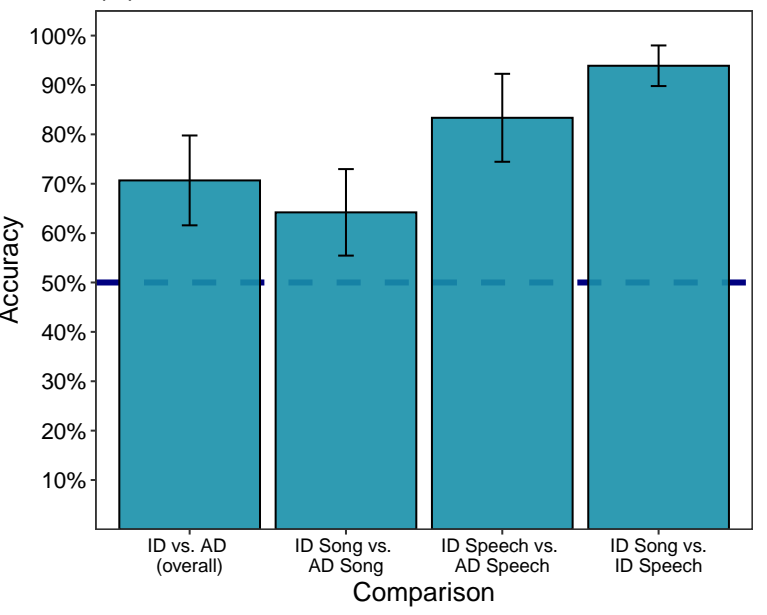

Figure 4. Accuracy of hypothesis-free classifiers. (A) The confusion matrix for the four-way categorical LASSO classifier shows successful classification in all four vocalization types. When misclassifying, the model is more likely to confuse the target (infant or adult) than the vocalization type (song or speech). (B) The bar graph displays the accuracy of each of the pairwise classifiers; all pairwise classifications were above chance level of $50 \%$ (denoted by the horizontal dotted line). Error bars denote $95 \%$ confidence intervals from corrected and re-sampled $t$-tests. Abbreviations: infant-directed (ID); adult-directed (AD).

The confusion matrix also reveals patterns of misidentification: in the $29.5 \%$ of recordings that are misidentified, the model rarely classifies songs as speech (or vice versa), but sometimes confuses the utterance target within the correct vocalization type. For example, infant-directed songs are more than 10 times more likely to be classified inaccurately as adult-directed songs than to be classified inaccurately as adult-directed speech - but nevertheless, the model accurately identifies them as infant-directed songs most of the time $(60.0 \%$ relative to chance level of $25 \%$ ).

To identify the acoustic features that most reliably differentiate pairs of vocalization types, we continued with a logistic LASSO classifier to test the same three pairwise comparisons as in the exploratory-confirmatory analyses and the analysis of the naïve listener experiment: (1) infant-directed vs. adult-directed vocalizations, overall; (2) infant-directed song vs. adult-directed song; and (3) infant-directed song vs. infant-directed speech. We also ran a fourth pairwise comparison, between infant-directed speech and adult-directed speech, as an exploratory analysis.

The classifiers performed strikingly well (Figure 4B; infant-directed vs. adult-directed vocalizations, overall: $70.7 \%$ [61.6\%, 79.8\%]; infant-directed song vs. adult-directed song: $64.2 \%$ [55.4\%, 73.0\%]; infant-directed song vs. infant-directed speech: $93.9 \%[89.8 \%, 98.0 \%])$. Infant-directed speech was also reliably distinguished from adult-directed speech $(83.4 \%[74.4 \%, 92.3 \%])$.

Last, we examined the acoustic features identified by the variable selection step of the LASSO procedure, which most reliably predict vocalization type across all 21 societies. These are reported in Table 2 .

There was substantial overlap between the results of the two approaches (Table 2): out of 31 features selected by the LASSO classifier, 22 were supported by at least one exploratory-confirmatory result, and of those, 6 were preregistered. Consistent with the exploratory-confirmatory analyses, the acoustic features that reliably distinguished between each vocalization form concerned pitch, formant, timbre, and temporal features; in some cases, these included additional variables, such as pulse clarity (the strength of the beats, detected via music information retrieval) and temporal modulation (the frequency decomposition of the amplitude envelope, or how quickly loudness changes). 


\begin{tabular}{|c|c|c|c|c|c|}
\hline Feature & Statistic & $\begin{array}{r}\text { ID }[+] \text { vs. AD } \\
{[-](\text { overall })} \\
\end{array}$ & $\begin{array}{l}\text { ID Song }[+] \text { vs. } \\
\text { AD Song }[-]\end{array}$ & $\begin{array}{l}\text { ID Song }[+] \text { vs. } \\
\text { ID Speech }[-]\end{array}$ & $\begin{array}{l}\text { ID Speech [+] vs. } \\
\text { AD Speech }[-]\end{array}$ \\
\hline \multirow[t]{2}{*}{ Attack Curve Slope } & IQR & 0.155 & 0.182 & 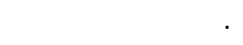 & 0.108 \\
\hline & Median $^{\text {pre }}$ & -0.139 & -0.373 & -0.352 & 0.176 \\
\hline Inharmonicity & Whole $^{\text {pre }}$ & -0.125 & -0.204 & -0.029 & -0.04 \\
\hline Pulse Clarity & Whole $^{\text {pre }}$ & 0.161 & 0.069 & 0.336 & 0.19 \\
\hline 85th Energy Percentile & Whole $^{\text {pre }}$ & -0.243 & -0.216 & . & -0.152 \\
\hline \multirow[t]{2}{*}{ Roughness } & IQR & -0.162 & -0.159 & -0.151 & \\
\hline & Median & 0.178 & . & -0.520 & 0.002 \\
\hline Tempo & Whole $^{\text {pre }}$ & . & 0.047 & 0.12 & -0.007 \\
\hline nPVI per Phrase & Whole $^{\text {pre }}$ & -0.053 & -0.061 & -0.021 & . \\
\hline \multirow[t]{2}{*}{ Pitch } & IQR & 0.093 & -0.16 & $\cdot$ & 0.386 \\
\hline & Median & 0.738 & 0.097 & 0.259 & 1.276 \\
\hline \multirow[t]{2}{*}{ Pitch Space } & IQR & -0.112 & -0.105 & -0.782 & . \\
\hline & Median & 0.108 & -0.216 & -0.909 & 0.128 \\
\hline \multirow[t]{2}{*}{ Pitch Rate } & IQR & 0.146 & -0.052 & -0.735 & 0.123 \\
\hline & Median & 0.178 & 0.306 & . & . \\
\hline \multirow[t]{3}{*}{ First Formant } & IQR & 0.032 & 0.024 & . & . \\
\hline & Median & -0.115 & -0.114 & -0.369 & . \\
\hline & Range & -0.23 & -0.328 & -0.121 & -0.009 \\
\hline Second Formant & Median & 0.042 & -0.149 & 0.082 & 0.176 \\
\hline \multirow[t]{2}{*}{ Intensity } & IQR & 0.471 & 0.295 & -0.225 & 0.456 \\
\hline & Median & -0.406 & -0.511 & 0.595 & \\
\hline \multirow[t]{2}{*}{ Intensity Space } & IQR & -0.72 & -0.543 & $\cdot$ & -0.523 \\
\hline & Median & -0.436 & -0.154 & -0.368 & -0.295 \\
\hline \multirow[t]{2}{*}{ Intensity Rate } & IQR & 0.466 & 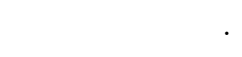 & . & 0.08 \\
\hline & Median & . & 0.6 & . & . \\
\hline \multirow[t]{2}{*}{ Vowel Space } & IQR & 0.51 & 0.911 & . & . \\
\hline & Median & 0.032 & 0.062 & $\cdot$ & . \\
\hline \multirow[t]{2}{*}{ Vowel Travel Rate } & IQR & 0.234 & 0.567 & . & . \\
\hline & Median & & -1.033 & -1.256 & 0.984 \\
\hline \multirow[t]{2}{*}{ Temporal Modulation } & Peak $^{\text {pre }}$ & 0.166 & 0.138 & . & . \\
\hline & $\mathrm{SD}^{\text {pre }}$ & 0.069 & 0.005 & 0.045 & 0.03 \\
\hline
\end{tabular}

Table 2. Acoustic features that reliably differentiate the four vocalization types, selected via LASSO classification with cross-validation across societies. The table reports coefficients from penalized logistic regressions using acoustic features (standardized within-voices). Changes in the values of the coefficients produce changes in the predicted log-odds ratio, so the values in the table can be interpreted as in a logistic regression. The features supported by convergent evidence from the exploratory-confirmatory analyses are in bold; those that were preregistered are marked with a superscript pre. Abbreviations: infant-directed (ID); adult-directed (AD). 


\subsection{Convergent analysis: Predicting listener intuitions from acoustic features}

Last, we examined the degree to which the naïve listener's perceptions of infant-directedness were explicable from the primary acoustic features identified by the exploratory-confirmatory and hypothesis-free analyses of the corpus. To reduce the risk of introducing false-positives in a large dataset, we only analyzed acoustic features that had convergent evidence from at least one summary statistic in both the exploratoryconfirmatory and LASSO analyses, in at least one comparison type. In these analyses, we collapsed across all vocalization types and attempted to predict only whether naïve listeners rated a given vocalization as infant- or adult-directed (regardless of society or vocalization type). This yielded 21 features. To justify a strong interpretation of potential relations between these 21 features and infant-directedness in the corpus, we regressed each vocalization's average infant-directedness score on each of the 21 features individually, using a strict Bonferroni-adjusted alpha level of .0024.

This procedure yielded 12 features that were significantly predictive of listeners' perceptions of infantdirectedness after this selection procedure (Figure 5 and Table S5). The most reliably associated feature, by far, was pitch: median $\mathrm{F}_{0}$ (Figure $5 \mathrm{~A}$ ) and its variability (Figure $5 \mathrm{~B}$ ) each accounted for about $30 \%$ of the variability in perceived infant-directedness; other features related to infant-directedness included intensity space (Figure 5C), temporal modulation (Figure 5D), roughness (Figure 5E), and inharmonicity (Figure 5F).

Last, we entered all 12 features into a multiple linear regression. These features explained $45.0 \%$ of the variability in perceived infant-directedness $(F(12,1081)=73.7, p<.0001)$. When entered into the regression together, 5 of the 12 features had significant partial effects (median $\mathrm{F}_{0}: \beta=0.30 ; \mathrm{F}_{0} \mathrm{IQR}: \beta=0.33$; median intensity travel rate: $\beta=-0.17$; roughness IQR: $\beta=-0.12$; median $\mathrm{F}_{1}: \beta=-0.09$ ). Thus, while 12 core acoustic features are reliably associated with infant-directedness across the corpus, there is nonetheless substantial additional variability in the infant-directedness of vocalizations that is left unexplained.

\section{Discussion}

We provide convergent evidence for widespread regularities in the acoustic design of infant-directed vocalizations, in both the domains of language and music. Naïve listeners reliably identified infant-directed vocalizations as infant-directed, despite the fact that the vocalizations were largely of unfamiliar geographic and linguistic origin, and more consistently in song than in speech. A series of hypothesis- and data-driven analyses showed consistent acoustic distinctions between infant-directed and adult-directed vocalizations overall, between infant-directed and adult-directed song, and between infant-directed song and infant-directed speech. These acoustic distinctions together explained nearly half the variability in listeners' perceptions of infant-directedness.

The most consistent ways in which infant-directed vocalizations differ from adult-directed vocalizations, worldwide, concern pitch: nearly every comparison revealed differences in pitch, pitch space, and pitch rate (Figure 3), and, moreover, $\mathrm{F}_{0}$ median and interquartile range explained by far the largest proportion of variability in listeners' perceived infant-directedness (Figure 5). But other acoustic features also reliably distinguished infant-directed vocalizations from adult-directed vocalizations, infant-directed song from adultdirected song, and infant-directed song from infant-directed speech - albeit in subtler ways that the LASSO classifier detected more reliably than did naïve listeners. These features included rhythmic, phonetic, and timbral characteristics of the vocalizations, such as temporal modulation, durational contrast, roughness, inharmonicity, and intensity space (Figure 4, Table 2, and Table S4).

Simply put: across many voices from many cultures producing many speech and song utterances, infantdirected vocalizations tend to sound different than adult-directed vocalizations. The differences are salient enough for naïve listeners to detect, because they are characterized by a core set of acoustic dimensions - more consistently in infant-directed song than in infant-directed speech. Taken together, these findings suggest a link between form and function in the design of infant-directed vocalizations.

Surprisingly, however, naïve listeners' intuitions about infant-directed speech were far less consistent across societies than their intuitions about infant-directed song. Corpus-wide, both vocalization types were iden- 
(A)

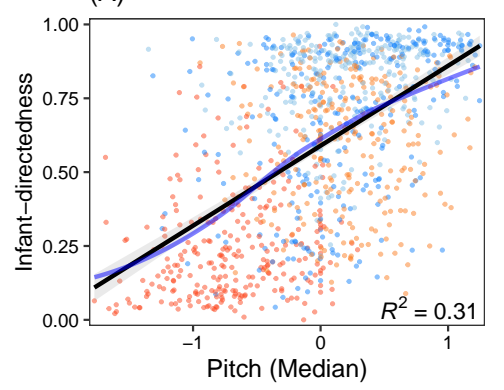

(D)



(G)

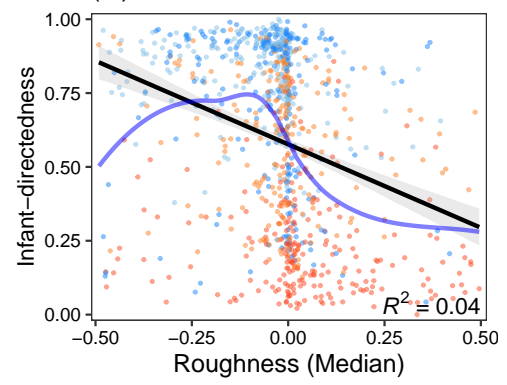

(J)

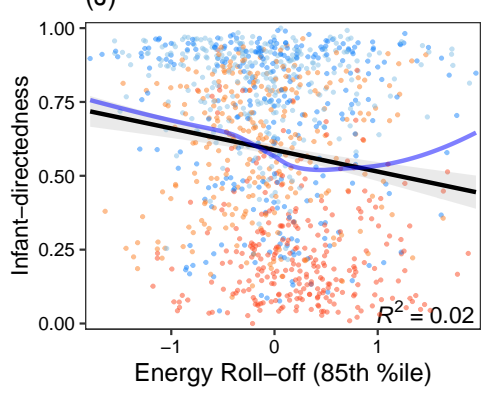

(B)

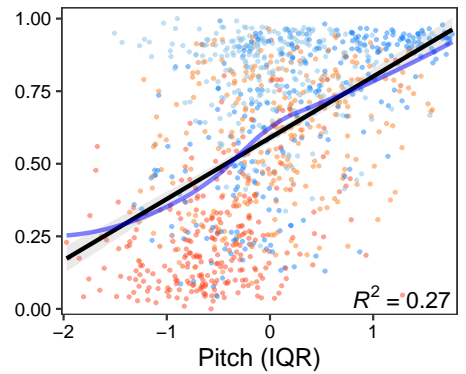

(E)



(H)

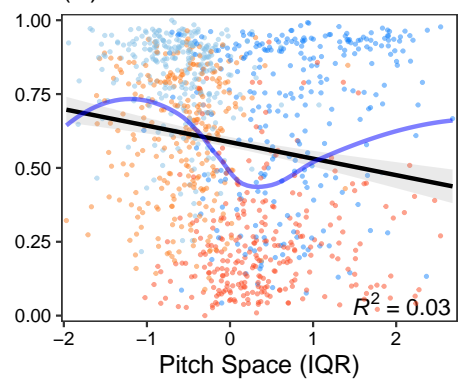

(K)

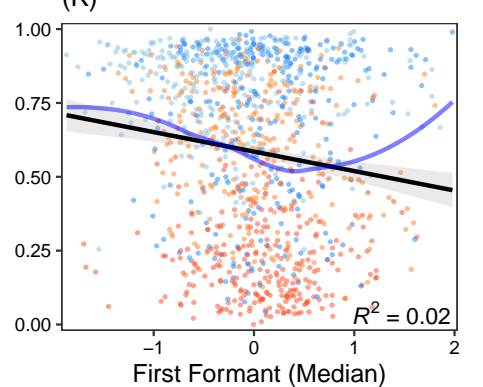

(C)

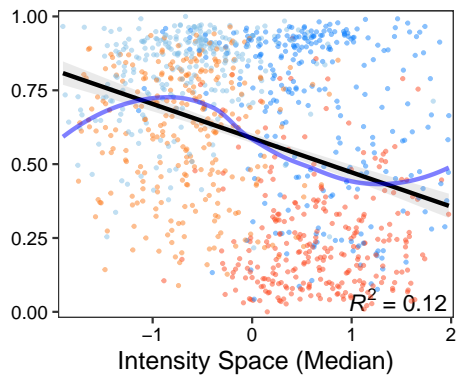

(F)
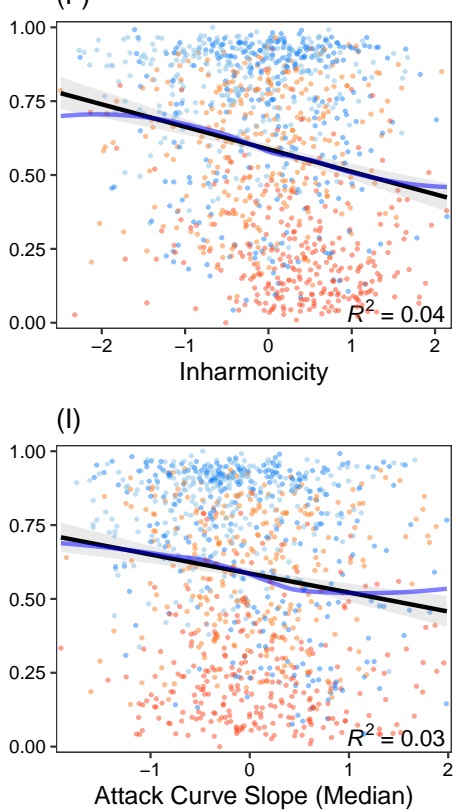

(L)

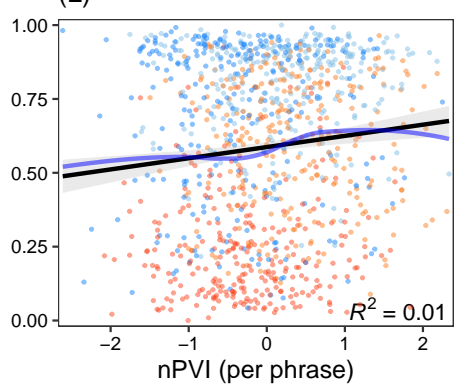

ID Song ID Speech AD Song AD Speech

Figure 5. Twelve acoustic features reliably predict infant-directedness across societies. (A-L) The scatterplots each correspond to a single acoustic feature (indicated on the $x$-axis). They represent the average naïve listener ratings of infant-directedness for each recording in the corpus (measured by the average proportion of "baby" responses in the online experiment), as a function of each acoustic feature (normalized across participants and centered within participants). The features plotted here survived a Bonferroni correction for 21 tests and, further, were included only if they were supported by convergent evidence from both LASSO and exploratory-confirmatory analyses. The black line represents the linear model corresponding to the reported $R^{2}$, which is significant at $p<.0024$; the gray shaded area a $95 \%$ confidence interval; and the blue line a LOESS regression. The $x$-axes of some panels are truncated to facilitate visualization. 
tified well above chance level, but this analysis masked some cross-cultural variability: when analyzing performance within each society independently, infant-directed song was always identified reliably, but infantdirected speech was identified reliably less than half the time. Moreover, those societies where the naïve listeners failed to identify infant-directed speech tended to be small-scale, contrasting with typical "citizen scientist" participants, who are recruited mostly from industrialized populations. This suggests that the corpus-wide identification rate for infant-directed speech is inflated by the listeners' familiarity with the style of infant-directed speech found in societies similar to their own - and raises the intruiging possibility that infant-directed speech is more variable, worldwide, than is infant-directed song.

This research leaves open at least four questions. First, while the results point in the direction of universality, because the corpus covers a swath of geographic locations (21 societies on 6 continents), languages (12 language families), and different subsistence regimes (8 types), the participants whose vocalizations we studied do not constitute a representative sample of humans. As such, no strong claims of universality are justified concerning the acoustic structure of infant-directedness. This issue could be addressed by (a) studying larger, representative samples of infant-directed vocalizations; (b) using phylogenetic methods to examine whether people in societies that are very distantly related nonetheless produce similar infantdirected vocalizations; (c) testing perceived infant-directedness in a more diverse sample of listeners, to more accurately characterize cross-cultural variability in the perception of infant-directedness; and (d) testing listener intuitions among groups with reduced exposure to a given set of infant-directed vocalizations, such as very young infants or people from distantly related small-scale societies.

Second, despite a large body of work in bioacoustics examining the structure of vocal signals ${ }^{1-3,3-15}$, it is not yet clear the extent to which the variability in acoustic features identified here is unique to humans, or whether it reflects more general principles underlying cross-species regularities in vocal signals. It is notable, for example, that many of the acoustic features that are reduced in infant-directed vocalization (Table 2) are associated with harsh, nonlinear sounds commonly accentuated in alarm calls across species ${ }^{4,78}$. Comparative studies may help to disentangle the ways in which human vocal signals are shaped in ways that are different from other animals, or not.

Third, our findings say little about the content of infant-directed vocalizations, which are known to vary widely: song and speech are used in a wide variety of contexts with infants, of which soothing (the type of vocalization we elicited from participants) is just one. One curious finding reported here, where naïve listeners reliably characterize adult-directed song inaccurately as infant-directed, may bear on this question - perhaps this simply reflects a predisposition in our listeners to finding solo, mostly female voices, as soothing - given a wider variety of contexts for the solo singing, perhaps the naïve listeners would have responded differently. Similarly, the sounds of arousing or alerting infant-directed speech and soothing infant-directed speech are likely to differ consistently from one another across cultures ${ }^{22}$, just as different forms of infant-directed song differ from one another (e.g., lullabies vs. play songs ${ }^{63}$ ). Future studies should determine the degree of generality of the present findings across a wider variety of contexts.

Last, the corpus-building approach used here may help to empirically test theories on the origins and functions of music and speech in infancy. For example, if infant-directed song communicates the costly investment of parental attention ${ }^{55}$, then infant-directed song should feature increased flashiness and variability in salient acoustic characteristics for infants - consistent with the present findings of higher energy in second formants (important for vowel recognition ${ }^{79}$ ) and faster travel over a vowel space. Moreover, the relation between infant-directedness and the sounds of vowels is consistent with classic experimental evidence demonstrating infants' robust perceptual sensitivity to vowels ${ }^{79-81}$. In contrast, cross-cultural variability in infant-directed speech found in the naïve listener experiment weighs against any universality prediction from functional accounts of infant-directed speech ${ }^{32-35}$; however, given the relatively high accuracy of the LASSO classifiers in distinguishing infant- from adult-directed speech across the societies studied, more research is needed to clarify those aspects of infant-directed speech that are culturally invariant.

Whatever the answers to these questions, the results presented here demonstrate that infant-directed vocalizations - and especially infant-directed song - are a fundamental aspect of human communication, characterized by acoustic regularities across many cultures. 


\section{Data, code, and materials availability}

Data and code are available at https://github.com/themusiclab/infant-vocal; the corpus is available at https://osf.io/m5yn2; the preregistration is at https://osf.io/5r72u; and readers may participate in the naïve listener experiment at https://themusiclab.org/quizzes/ids.

\section{Author contributions}

S.A.M. and M.M.K. conceived of the research, provided funding, and coordinated the recruitment of collaborators and creation of the corpus. L.G., A.G., G.J., C.T.R., M.B.N., A.M., L.K.C., S.E.T., J. Song, M.K., A.S., T.A.V., Q.D.A., J.A., P.M., A.S., C.D.P., G.D.S., S.K., M.S., S.A.C., J.Q.P., C.S., J. Stieglitz, C.M., R.R.S., and B.M.W. collected the field recordings. C.M.B. and S.A. provided essential research assistance. S.A.M., C.M.B., and J. Simson designed and implemented the online experiment. C.J.M. and H.L-R. processed all recordings and designed the acoustic feature extraction in collaboration with S.A.M. and M.M.K. S.A.M. led analyses, with contributions from C.J.M., D.K., and M.M.K. S.A.M. made the figures. C.J.M., H.L-R., M.M.K., and S.A.M. wrote the manuscript and all authors approved it.

\section{Ethics}

Informed consent was obtained from all participants. Ethics approval for the naïve listener experiment was provided by the Committee on the Use of Human Subjects, Harvard University's Insitutional Review Board (protocol \#IRB17-1206). Ethics approval for the collection of recordings and their use in research was decentralized; each collaborating research arranged ethics approval with their local institution.

\section{Competing interests}

We declare we have no competing interests.

\section{Funding}

This research was supported by the Harvard University Department of Psychology (M.M.K. and S.A.M.); the Harvard College Research Program (H.L-R.); the Harvard Data Science Initiative (S.A.M.); the National Institutes of Health Director's Early Independence Award DP5OD024566 (S.A.M.); the Academy of Finland Grant 298513 (J.A.); the Royal Society of New Zealand Te Apārangi Rutherford Discovery Fellowship RDFUOA1101 (Q.D.A., T.A.V.); the Social Sciences and Humanities Research Council of Canada (L.K.C.); the Polish Ministry of Science and Higher Education grant N43/DBS/000068 (G.J.); the Fogarty International Center and National Heart, Lung, and Blood Institute, and the National Institute of Neurological Disorders and Stroke Award D43 TW010540 (P.M., C.D.P.); the National Institute of Allergy and Infectious Diseases Award R15-AI128714-01 (P.M.); the Max Planck Institute for Evolutionary Anthropology (C.T.R.); a British Academy Research Fellowship and Grant SRG-171409 (G.D.S.); the Institute for Advanced Study in Toulouse, under an Agence nationale de la recherche grant, Investissements d'Avenir ANR-17-EURE-0010 (J. Stieglitz); and the Natural Sciences and Engineering Research Council of Canada (S.E.T.).

\section{Acknowledgments}

We thank the participants and their families for providing recordings; D. Amir, who sparked the idea for this research in conversation with S.A.M. at the 2016 Annual Conference of the Human Behavior \& Evolution Society; J. Du, E. Pillsworth, L. Sugiyama, P. Wiessner, and J. Ziker, who collected or attempted to collect additional recordings; A. Bergson, Z. Jurewicz, D. Li, L. Lopez, and E. Radytè for research assistance; and M. Bertolo, J. Kominsky, and L. Yurdum for feedback on the manuscript. 


\section{Supplementary Information}

\section{Details of acoustic feature extraction}

\section{Praat}

We extracted intensity, pitch, and first and second formant values from the denoised recordings every 0.03125 seconds. For male participants, the pitch floor was set at $75 \mathrm{~Hz}$, with a pitch ceiling at $300 \mathrm{~Hz}$, and a maximum formant of $5000 \mathrm{~Hz}$. For females these values were $100 \mathrm{~Hz}, 600 \mathrm{~Hz}$, and $5500 \mathrm{~Hz}$, respectively. From these data, several summary values were calculated per recording: mean and maximum first and second formants, mean pitch, and minimum intensity. In addition to these summary statistics, we measured the intensity and pitch rates as change in these values over time. For vowel measures, the first and second formants were used to calculate both the average vowel space used, as well as the vowel change rate (measured as change in Euclidean formant space) over time.

\section{MIRtoolbox}

All MIRtoolbox (v. 1.7.2) features were extracted with default parameters ${ }^{69}$. mirattackslope returns a list of all attack slopes detected, so final analyses were done on summary features (e.g., mean, median, etc.). Final analyses were also done on summary features for mirroughness, which returns time series data of roughness measures in 50ms windows. We RMS-normalized the mean of mirroughness following ${ }^{82}$. MIRtoolbox features were computed on the denoised recordings, with the exception of mirtempo and mirpulseclarity, where removing the silences between vocalizations would have altered the tempo.

\section{Rhythmic variability}

For temporal modulation spectra we followed Ding's ${ }^{83}$ method, which combines discrete Fourier transforms applied to contiguous six-second excerpts. To analyze the entirety of each recording, we appended all recordings with silence to be exact multiples of six-seconds. The location of the peak $(\mathrm{Hz})$ and variance of the temporal modulation spectra were extracted from their RMS values.

\section{Normalized pairwise variability index}

The nPVI represents the temporal variance of data with discrete events, which makes it especially useful for comparing speech and music ${ }^{70}$. We used an automated syllable- and phrase-detection algorithm to extract events $^{71}$. We computed nPVI in two ways: by averaging the nPVI of each phrase within a recording, as well as by treating the entire recording as a single phrase. Because intervening silence would influence both temporal modulation and nPVI measures, we used recordings before they had been denoised. 
bioRxiv preprint doi: https://doi.org/10.1101/2020.04.09.032995; this version posted April 11, 2020. The copyright holder for this preprint (which

was not certified by peer review) is the author/funder, who has granted bioRxiv a license to display the preprint in perpetuity. It is made available under aCC-BY-NC-ND 4.0 International license.

Who's Listening?

Someone is speaking or singing. Who do you think they are singing or speaking to?

Press $\mathbf{F}$ for adult or $\mathbf{J}$ for baby.

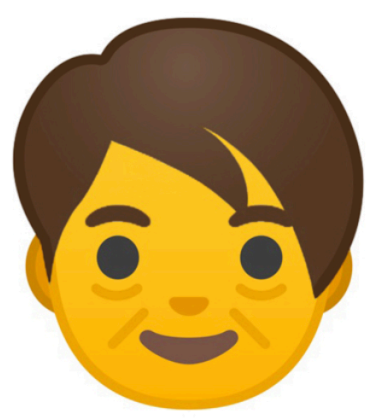

$\mathbf{F}$

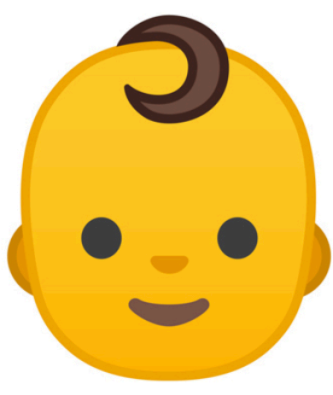

$\mathbf{J}$

Try to answer as quickly as you can!

Figure S1. Screenshot from the naïve listener experiment (desktop computer version). On each trial, participants heard a randomly selected vocalization from the corpus and were asked to quickly guess to whom the vocalization was directed: an adult or an infant. 


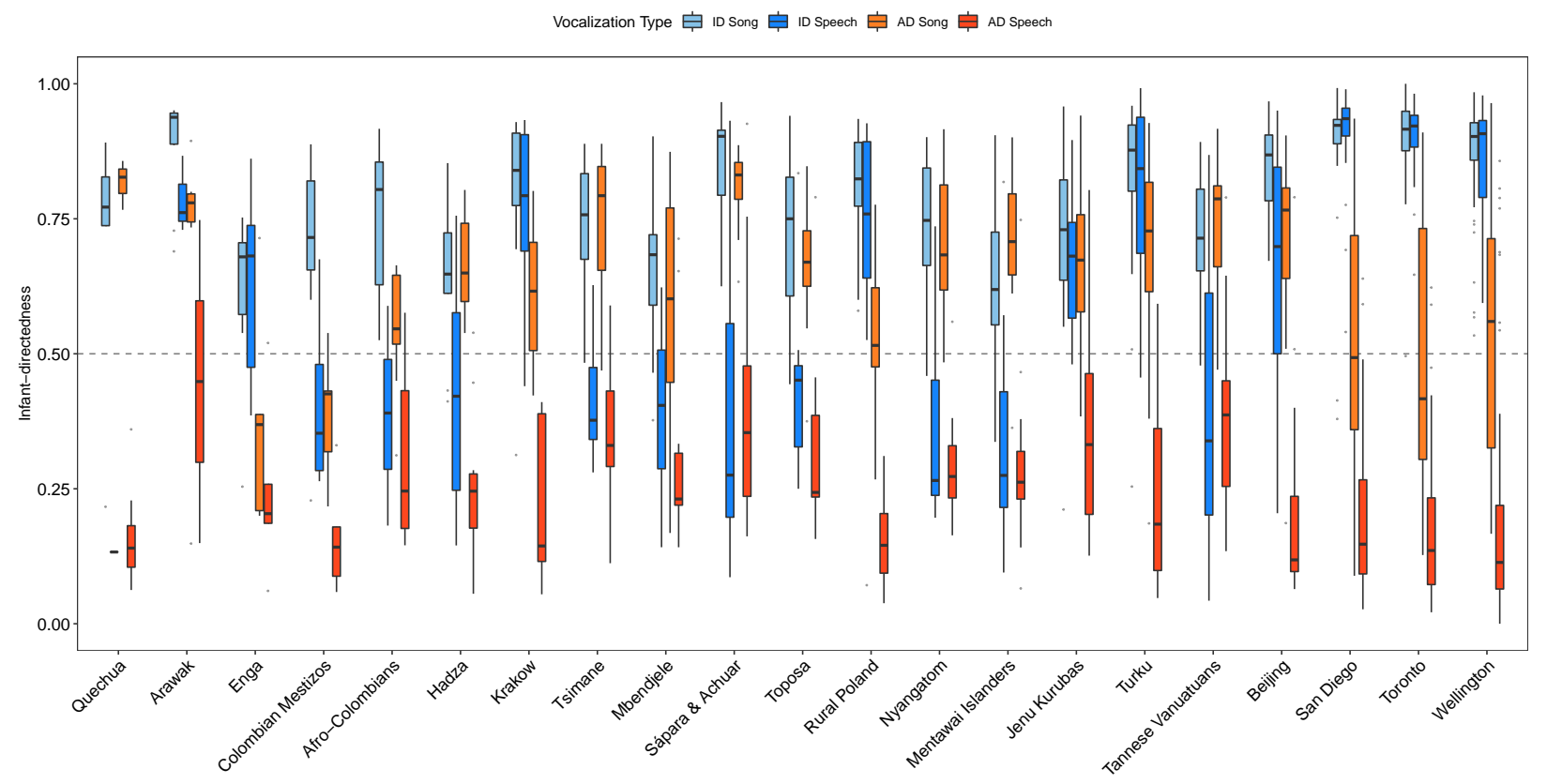

Figure S2. Perceived infant-directedness, analyzed separately for each society. For each vocalization type, the boxplots indicate the within-society median (horizontal black line), interquartile range (box), $1.5 \times$ IQR (whiskers), and outliers (gray points). The societies are ordered from the smallest to largest number of recordings (from left to right). Abbreviations: infant-directed (ID); adult-directed (AD). 


\begin{tabular}{|c|c|c|}
\hline Variable & Label & Description \\
\hline id & filename & \\
\hline mir_attack & Attack Curve Slope & $\begin{array}{l}\text { MIRtoolbox detects events in the audio; for a subset of those it can compute an } \\
\text { attack slope, which is the slope of the line from the beginning of the event to its } \\
\text { peak. }\end{array}$ \\
\hline mir_roughness & Roughness & $\begin{array}{l}\text { A roughness measure based on the dissonant beating patterns produced by } \\
\text { interference frequencies in the spectrum of the sound. MIRtoolbox produces a } \\
\text { roughness curve; following Buyens et al. (2017), we reduce this to a single measure } \\
\text { by taking the RMS-normalized mean. }\end{array}$ \\
\hline mir_rolloff85 & $\begin{array}{l}\text { 85th Energy } \\
\text { Percentile }\end{array}$ & $\begin{array}{l}\text { One way to estimate the amount of high frequency in the signal consists in finding } \\
\text { the frequency such that a certain fraction of the total energy is contained below } \\
\text { that frequency. This ratio is fixed by default to } .85 \text { (following Tzanetakis and } \\
\text { Cook, 2002), other have proposed } .95 \text { (Pohle, Pampalk and Widmer, 2005). }\end{array}$ \\
\hline mir_inharmonicity & Inharmonicity & $\begin{array}{l}\text { mirinharmonicity "estimates the inharmonicity, i.e., the amount of partials that } \\
\text { are not multiples of the [automatically detected] fundamental frequency, as a value } \\
\text { between } 0 \text { and } 1 . \text { More precisely, the inharmonicity considered here takes into } \\
\text { account the amount of energy outside the ideal harmonic series." (MIRtoolbox } \\
\text { manual) }\end{array}$ \\
\hline mir_tempo & Tempo & $\begin{array}{l}\text { MIRtoolbox tempo detection with default parameters. Based on MIRtoolbox's } \\
\text { event detection. Outputs a single number. }\end{array}$ \\
\hline mir_pulseclarity & Pule Clarity & $\begin{array}{l}\text { Estimates the rhythmic clarity, indicating the strength of the beats estimated by } \\
\text { the mirtempo function. }\end{array}$ \\
\hline npvi_total & nPVI Recording & $\begin{array}{l}\text { The nPVI equation measures the "average degree of durational contrast between } \\
\text { adjacent events in a sequence" (Daniele \& Patel, 2015). This makes it especially } \\
\text { useful for comparing rhythmic units across language and music (i.e., syllables vs. } \\
\text { notes). To automatically detect events, we used Mertens' (2004) syllable detection } \\
\text { algorithm. }\end{array}$ \\
\hline npvi_phrase & nPVI Phrase & $\begin{array}{l}\text { In addition to detecting syllables, Mertens' algorithm detects phrases. Whereas } \\
\text { npvi_total computes nPVI based on the whole file as a continuous phrase, this } \\
\text { measure computes the nPVI for each detected phrase and reports the mean. In } \\
\text { other words, it excludes the distances between the ends and beginnings of phrases. }\end{array}$ \\
\hline tm_std_hz & $\begin{array}{l}\text { Temporal } \\
\text { Modulation }\end{array}$ & $\begin{array}{l}\text { The temporal modulations spectrum is the frequency decomposition of the } \\
\text { amplitude envelope of a signal. This measures how loud something is at any given } \\
\text { moment, and then we measure how fast the loudness changes. Trivial example: if } \\
\text { the song is someone singing a note every second, the spectrum will have a peak at } \\
1 \mathrm{~Hz} \text {. If the song is someone singing a note three times a second, but with an } \\
\text { emphasis every three seconds, there will be a large peak at } 1 \mathrm{~Hz} \text {, and a smaller } \\
\text { peak at } 3 \mathrm{~Hz} \text {. We're interested in the standard deviation of the spectrum, which } \\
\text { we're construing as how exaggerated the peak is. }\end{array}$ \\
\hline praat_f0 & Pitch & The pitch (f0) in Hertz for each song \\
\hline praat_pitch_rate & Pitch Rate & $\begin{array}{l}\text { The pitch rate is a measure of pitch change over unit time. In essence, the pitch } \\
\text { rate gives us a measure of pitch curve smoothness (a lower value corresponds to a } \\
\text { smoother curve). }\end{array}$ \\
\hline praat_vowtrav & Vowel Space & $\begin{array}{l}\text { The euclidian distance travelled in vowel space. This is equivalent to distance } \\
\text { between two formants. }\end{array}$ \\
\hline praat_vowtrav_rate & $\begin{array}{l}\text { Vowel Space Travel } \\
\text { Rate }\end{array}$ & $\begin{array}{l}\text { The euclidian distance travelled in vowel space over a rate of time. This is } \\
\text { equivalent to distance between two formants divided by rate of travel. }\end{array}$ \\
\hline praat_intensity & Amplitude & A measure of amplitude (loudness) in decibels \\
\hline praat_intensity_rate & Amplitude Rate & $\begin{array}{l}\text { A measure of decay in intensity curves in each song measured as change in } \\
\text { intensity over rate in time. }\end{array}$ \\
\hline praat_f1 & First Formant & The frequency in Herz of the first formant at each $(.03125 / \mathrm{sec})$ point \\
\hline praat_f2 & Second Formant & The frequency in Herz of the second formant at each $(.03125 / \mathrm{sec})$ point \\
\hline meta_length & File duration & The length of the unedited sound files \\
\hline meta_edit_length & $\begin{array}{l}\text { Concatenated file } \\
\text { duration }\end{array}$ & The length of the concatenated versions of the sound files \\
\hline
\end{tabular}

Table S1. Codebook for acoustic features. Variable names are stubs, i.e., in the datasets, suffixes are added to denote summary statistics. Abbreviations: infant-directed (ID); adult-directed (AD). 
bioRxiv preprint doi: https://doi.org/10.1101/2020.04.09.032995; this version posted April 11, 2020. The copyright holder for this preprint (which

was not certified by peer review) is the author/funder, who has granted bioRxiv a license to display the preprint in perpetuity. It is made available under aCC-BY-NC-ND 4.0 International license.

\begin{tabular}{|c|c|c|c|c|c|c|}
\hline Comparison & Feature & Statistic & Est. & $S E$ & $z$ & $p$ \\
\hline \multirow{32}{*}{$\begin{array}{l}\text { ID vs. AD } \\
\text { (overall) }\end{array}$} & $\begin{array}{l}\text { 85th Energy } \\
\text { Percentile }\end{array}$ & Whole & -744.65 & 155.62 & -4.79 & $<.001$ \\
\hline & Attack Curve Slope & Median & 0.41 & 0.20 & 2.03 & 0.043 \\
\hline & \multirow[t]{2}{*}{ First Formant } & Maximum & -172.06 & 35.97 & -4.78 & $<.001$ \\
\hline & & Range & -186.41 & 38.91 & -4.79 & $<.001$ \\
\hline & Inharmonicity & Whole & -0.01 & 0.00 & -4.28 & $<.001$ \\
\hline & \multirow[t]{2}{*}{ Intensity } & IQR & 0.68 & 0.30 & 2.22 & 0.026 \\
\hline & & Minimum & 0.86 & 0.38 & 2.27 & 0.023 \\
\hline & \multirow[t]{2}{*}{ Intensity Rate } & Whole & -4.42 & 0.48 & -9.25 & $<.001$ \\
\hline & & Whole & 2.99 & 0.43 & 6.92 & $<.001$ \\
\hline & \multirow[t]{2}{*}{ Intensity Space } & Mean & 0.62 & 0.11 & 5.79 & $<.001$ \\
\hline & & St. Dev. & 1.76 & 0.26 & 6.65 & $<.001$ \\
\hline & \multirow{7}{*}{ Pitch } & First Quartile & 27.88 & 4.04 & 6.91 & $<.001$ \\
\hline & & Third Quartile & 59.44 & 11.28 & 5.27 & $<.001$ \\
\hline & & IQR & 31.52 & 8.55 & 3.69 & $<.001$ \\
\hline & & Mean & 42.19 & 6.91 & 6.11 & $<.001$ \\
\hline & & Median & 45.47 & 7.34 & 6.19 & $<.001$ \\
\hline & & Minimum & 8.13 & 2.72 & 2.99 & 0.003 \\
\hline & & St. Dev. & 13.00 & 3.64 & 3.57 & $<.001$ \\
\hline & \multirow[t]{2}{*}{ Pitch Rate } & Whole & -37.30 & 4.34 & -8.59 & $<.001$ \\
\hline & & Whole & 23.36 & 4.62 & 5.05 & $<.001$ \\
\hline & \multirow[t]{4}{*}{ Pitch Space } & First Quartile & 0.51 & 0.10 & 5.18 & $<.001$ \\
\hline & & Mean & 3.24 & 1.34 & 2.42 & 0.015 \\
\hline & & Median & 1.61 & 0.33 & 4.87 & $<.001$ \\
\hline & & St. Dev. & 6.99 & 2.35 & 2.98 & 0.003 \\
\hline & \multirow[t]{3}{*}{ Second Formant } & Maximum & -114.81 & 25.77 & -4.46 & $<.001$ \\
\hline & & Median & 35.63 & 12.51 & 2.85 & 0.004 \\
\hline & & Range & -115.51 & 33.30 & -3.47 & 0.001 \\
\hline & \multirow[t]{4}{*}{ Vowel Space } & Third Quartile & 46.81 & 15.12 & 3.10 & 0.002 \\
\hline & & IQR & 45.23 & 12.97 & 3.49 & $<.001$ \\
\hline & & Mean & 38.13 & 10.68 & 3.57 & $<.001$ \\
\hline & & St. Dev. & 51.71 & 10.80 & 4.79 & $<.001$ \\
\hline & Vowel Space Travel & Whole & 212.31 & 37.85 & 5.61 & $<.001$ \\
\hline \multirow{19}{*}{$\begin{array}{l}\text { ID Song vs. AD } \\
\text { Song }\end{array}$} & \multirow[t]{2}{*}{ Attack Curve Slope } & First Quartile & -0.45 & 0.21 & -2.12 & 0.034 \\
\hline & & Median & -0.80 & 0.41 & -1.97 & 0.049 \\
\hline & First Formant & Median & -19.66 & 9.70 & -2.03 & 0.043 \\
\hline & \multirow{7}{*}{$\begin{array}{l}\text { Inharmonicity } \\
\text { Intensity }\end{array}$} & Whole & -0.01 & 0.00 & -2.15 & 0.032 \\
\hline & & First Quartile & -1.95 & 0.55 & -3.57 & $<.001$ \\
\hline & & Third Quartile & -1.45 & 0.50 & -2.88 & 0.004 \\
\hline & & Maximum & -1.13 & 0.51 & -2.22 & 0.027 \\
\hline & & Mean & -1.60 & 0.48 & -3.35 & 0.001 \\
\hline & & Median & -1.63 & 0.51 & -3.18 & 0.001 \\
\hline & & Minimum & -0.80 & 0.31 & -2.59 & 0.01 \\
\hline & \multirow{3}{*}{$\begin{array}{l}\text { nPVI Recording } \\
\text { Pitch } \\
\text { Tempo }\end{array}$} & Whole & -2.14 & 0.86 & -2.50 & 0.012 \\
\hline & & Minimum & -9.00 & 3.00 & -3.00 & 0.003 \\
\hline & & Whole & 5.80 & 2.75 & 2.11 & 0.035 \\
\hline & \multirow{5}{*}{$\begin{array}{l}\text { Temporal } \\
\text { Modulation } \\
\text { Vowel Space }\end{array}$} & Peak & 0.65 & 0.32 & 2.03 & 0.042 \\
\hline & & Third Quartile & 27.90 & 11.00 & 2.54 & 0.011 \\
\hline & & IQR & 24.94 & 9.58 & 2.60 & 0.009 \\
\hline & & Mean & 20.29 & 6.74 & 3.01 & 0.003 \\
\hline & & St. Dev. & 18.94 & 6.31 & 3.00 & 0.003 \\
\hline & Vowel Space Travel & Whole & 23.44 & 11.22 & 2.09 & 0.037 \\
\hline ID Song vs. ID & $\begin{array}{l}\text { Rate } \\
\text { Attack Curve Slope }\end{array}$ & First Quartile & -0.67 & 0.26 & -2.59 & 0.01 \\
\hline
\end{tabular}




\begin{tabular}{|c|c|c|c|c|c|c|}
\hline Comparison & Feature & Statistic & Est. & $S E$ & $z$ & $p$ \\
\hline & \multirow{11}{*}{ First Formant } & Third Quartile & -1.85 & 0.37 & -5.05 & $<.001$ \\
\hline & & IQR & -1.19 & 0.27 & -4.39 & $<.001$ \\
\hline & & Mean & -1.11 & 0.27 & -4.13 & $<.001$ \\
\hline & & Median & -1.17 & 0.32 & -3.65 & $<.001$ \\
\hline & & First Quartile & -24.19 & 7.30 & -3.31 & 0.001 \\
\hline & & Third Quartile & -57.27 & 19.77 & -2.90 & 0.004 \\
\hline & & Maximum & 112.08 & 30.98 & 3.62 & $<.001$ \\
\hline & & Mean & -39.98 & 10.94 & -3.66 & $<.001$ \\
\hline & & Median & -41.69 & 11.88 & -3.51 & $<.001$ \\
\hline & & Minimum & -26.11 & 5.25 & -4.97 & $<.001$ \\
\hline & & Range & 138.18 & 34.18 & 4.04 & $<.001$ \\
\hline & \multirow{2}{*}{$\begin{array}{l}\text { Inharmonicity } \\
\text { Intensity }\end{array}$} & Whole & -0.01 & 0.00 & -3.10 & 0.002 \\
\hline & & First Quartile & 1.29 & 0.48 & 2.68 & 0.007 \\
\hline & \multirow{5}{*}{ Intensity Space } & IQR & -1.44 & 0.35 & -4.15 & $<.001$ \\
\hline & & Minimum & -0.94 & 0.35 & -2.64 & 0.008 \\
\hline & & St. Dev. & -0.60 & 0.20 & -2.92 & 0.003 \\
\hline & & First Quartile & -0.29 & 0.03 & -9.56 & $<.001$ \\
\hline & & Third Quartile & -1.68 & 0.23 & -7.30 & $<.001$ \\
\hline & \multirow[b]{5}{*}{ nPVI Phrase } & IQR & -1.39 & 0.20 & -6.80 & $<.001$ \\
\hline & & Mean & -1.73 & 0.15 & -11.74 & $<.001$ \\
\hline & & Median & -0.76 & 0.08 & -9.48 & $<.001$ \\
\hline & & St. Dev. & -2.66 & 0.29 & -9.08 & $<.001$ \\
\hline & & Whole & 7.21 & 1.27 & 5.67 & $<.001$ \\
\hline & \multirow{3}{*}{$\begin{array}{l}\text { nPVI Recording } \\
\text { Pitch }\end{array}$} & Whole & 5.68 & 1.34 & 4.25 & $<.001$ \\
\hline & & Maximum & -23.98 & 11.46 & -2.09 & 0.036 \\
\hline & & St. Dev. & -11.25 & 5.10 & -2.21 & 0.027 \\
\hline & \multirow[t]{2}{*}{ Pitch Space } & First Quartile & -0.54 & 0.16 & -3.38 & 0.001 \\
\hline & & Third Quartile & -14.25 & 1.78 & -8.02 & $<.001$ \\
\hline & \multirow{10}{*}{$\begin{array}{l}\text { Pulse Clarity } \\
\text { Roughness }\end{array}$} & IQR & -13.71 & 1.81 & -7.57 & $<.001$ \\
\hline & & Maximum & -23.15 & 11.48 & -2.02 & 0.044 \\
\hline & & Mean & -16.16 & 1.50 & -10.76 & $<.001$ \\
\hline & & Median & -2.97 & 0.31 & -9.70 & $<.001$ \\
\hline & & Range & -23.15 & 11.48 & -2.02 & 0.044 \\
\hline & & St. Dev. & -18.79 & 2.56 & -7.35 & $<.001$ \\
\hline & & Whole & 0.02 & 0.01 & 3.44 & 0.001 \\
\hline & & Third Quartile & -13.00 & 3.99 & -3.26 & 0.001 \\
\hline & & Distance & -746.17 & 224.00 & -3.33 & 0.001 \\
\hline & & IQR & -12.96 & 3.91 & -3.32 & 0.001 \\
\hline & \multirow{5}{*}{ Second Formant } & Mean & -177.13 & 41.50 & -4.27 & $<.001$ \\
\hline & & Median & -2.55 & 0.96 & -2.66 & 0.008 \\
\hline & & St. Dev. & -54.89 & 18.84 & -2.91 & 0.004 \\
\hline & & Maximum & 83.42 & 27.09 & 3.08 & 0.002 \\
\hline & & Median & -49.14 & 21.99 & -2.23 & 0.025 \\
\hline & \multirow{5}{*}{$\begin{array}{l}\text { Temporal } \\
\text { Modulation } \\
\text { Vowel Space }\end{array}$} & Minimum & -69.20 & 23.20 & -2.98 & 0.003 \\
\hline & & Range & 152.58 & 41.31 & 3.69 & $<.001$ \\
\hline & & St. Dev. & 0.53 & 0.06 & 8.23 & $<.001$ \\
\hline & & First Quartile & -24.33 & 3.59 & -6.77 & $<.001$ \\
\hline & & Third Quartile & -97.33 & 14.50 & -6.71 & $<.001$ \\
\hline & \multirow[b]{5}{*}{$\begin{array}{l}\text { Vowel Space Travel } \\
\text { Rate }\end{array}$} & IQR & -73.02 & 11.76 & -6.21 & $<.001$ \\
\hline & & Mean & -82.31 & 9.28 & -8.87 & $<.001$ \\
\hline & & Median & -47.59 & 6.97 & -6.83 & $<.001$ \\
\hline & & St. Dev. & -83.54 & 10.56 & -7.91 & $<.001$ \\
\hline & & Whole & -298.47 & 32.34 & -9.23 & $<.001$ \\
\hline
\end{tabular}

Table S2. Significant results from exploratory analyses, using post-hoc linear combinations following multi-level mixedeffects models. Abbreviations: infant-directed (ID); adult-directed (AD). 


\begin{tabular}{|c|c|c|c|c|}
\hline Feature & Variable & ID vs. $\mathrm{AD}$ & $\begin{array}{l}\text { ID Song vs. AD } \\
\text { Song }\end{array}$ & $\begin{array}{l}\text { ID Song vs. ID } \\
\text { Speech }\end{array}$ \\
\hline $\begin{array}{l}\text { 85th Energy } \\
\text { Percentile }\end{array}$ & Whole & $-^{1}$ & - & - \\
\hline $\begin{array}{l}\text { Attack Curve } \\
\text { Slopes }\end{array}$ & Median & - & -1 & $-{ }^{1}$ \\
\hline $\begin{array}{l}\text { Attack Curve } \\
\text { Slopes }\end{array}$ & Mean & - & - & $--^{1}$ \\
\hline First Formant & Mean & - & - & -1 \\
\hline First Formant & $\operatorname{Max}$ & -1 & - & $-{ }^{0}$ \\
\hline Inharmonicity & Whole & -1 & - & -1 \\
\hline Intensity & Minimum & -1 & - & -1 \\
\hline Intensity Rate & Whole & $-^{0}$ & - & -1 \\
\hline nPVI per Phrase & Whole & + & + & $+^{1}$ \\
\hline $\begin{array}{l}\text { nPVI per } \\
\text { Recording }\end{array}$ & Whole & + & + & $+^{1}$ \\
\hline Pitch & Mean & $+^{1}$ & - & - \\
\hline Pitch Space & Mean & - & - & -1 \\
\hline Pitch Rate & Whole & -0 & - & $-{ }^{1}$ \\
\hline Pulse Clarity & Whole & $+^{1}$ & + & + \\
\hline Roughness & Mean & - & - & -1 \\
\hline Second Formant & Mean & - & - & - \\
\hline Second Formant & $\operatorname{Max}$ & $-{ }^{1}$ & $-^{0}$ & $-{ }^{0}$ \\
\hline Tempo & Whole & - & - & - \\
\hline $\begin{array}{l}\text { Temporal } \\
\text { Modulation }\end{array}$ & St. Dev. & - & - & $-{ }^{0}$ \\
\hline $\begin{array}{l}\text { Temporal } \\
\text { Modulation }\end{array}$ & Peak & $-^{0}$ & - & $-{ }^{1}$ \\
\hline Vowel Space & Mean & $+^{1}$ & $+^{1}$ & + \\
\hline $\begin{array}{l}\text { Vowel Space } \\
\text { Travel Rate }\end{array}$ & Whole & $++^{1}$ & $+{ }^{1}$ & + \\
\hline
\end{tabular}

Table S3. $\quad$ Preregistered predictions. Predictions that were supported by the exploratory-confirmatory analyses are marked ${ }^{1}$ while predictions which were significantly falsified in the opposite direction are marked ${ }^{0}$. Abbreviations: infant-directed (ID); adult-directed (AD). 
bioRxiv preprint doi: https://doi.org/10.1101/2020.04.09.032995; this version posted April 11, 2020. The copyright holder for this preprint (which

was not certified by peer review) is the author/funder, who has granted bioRxiv a license to display the preprint in perpetuity. It is made available under aCC-BY-NC-ND 4.0 International license.

\begin{tabular}{|c|c|c|c|c|c|c|}
\hline Comparison & Feature & Statistic & Est. & $S E$ & $z$ & $p$ \\
\hline \multirow{17}{*}{$\begin{array}{l}\text { ID vs. } \mathrm{AD} \\
\text { (overall) }\end{array}$} & 85th Energy & Whole & -665.11 & 182.20 & -3.65 & $<.001$ \\
\hline & Percentile & & & & & \\
\hline & Inharmonicity & Whole & -0.01 & 0.00 & -3.03 & 0.002 \\
\hline & Intensity & IQR & 0.46 & 0.18 & 2.51 & 0.012 \\
\hline & \multirow[t]{3}{*}{ Intensity Rate } & Whole & 2.07 & 0.43 & 4.81 & $<.001$ \\
\hline & & IQR & 2.08 & 0.52 & 4.04 & $<.001$ \\
\hline & & Median & 0.85 & 0.21 & 4.05 & $<.001$ \\
\hline & \multirow[t]{2}{*}{ Pitch } & IQR & 26.27 & 5.89 & 4.46 & $<.001$ \\
\hline & & Median & 41.55 & 7.64 & 5.44 & $<.001$ \\
\hline & \multirow[t]{3}{*}{ Pitch Rate } & Whole & 13.20 & 3.30 & 4.00 & $<.001$ \\
\hline & & IQR & 12.61 & 3.29 & 3.84 & $<.001$ \\
\hline & & Median & 3.12 & 0.66 & 4.70 & $<.001$ \\
\hline & \multirow{4}{*}{$\begin{array}{l}\text { Pitch Space } \\
\text { Vowel Space } \\
\text { Vowel Space Travel } \\
\text { Rate }\end{array}$} & Median & 1.19 & 0.25 & 4.73 & $<.001$ \\
\hline & & IQR & 30.83 & 12.52 & 2.46 & 0.014 \\
\hline & & Whole & 144.97 & 29.12 & 4.98 & $<.001$ \\
\hline & & IQR & 179.47 & 41.49 & 4.33 & $<.001$ \\
\hline & & Median & 71.18 & 15.08 & 4.72 & $<.001$ \\
\hline \multirow{8}{*}{$\begin{array}{l}\text { ID Song vs. AD } \\
\text { Song }\end{array}$} & Attack Curve Slope & Median & -0.44 & 0.19 & -2.31 & 0.021 \\
\hline & First Formant & Median & -12.58 & 6.02 & -2.09 & 0.037 \\
\hline & Intensity & IQR & -1.73 & 0.24 & -7.17 & $<.001$ \\
\hline & & Median & -1.20 & 0.54 & -2.22 & 0.026 \\
\hline & Vowel Space & IQR & 26.84 & 7.02 & 3.82 & $<.001$ \\
\hline & Vowel Space Travel & Whole & 24.82 & 11.50 & 2.16 & 0.031 \\
\hline & Rate & IQR & 39.07 & 16.13 & 2.42 & 0.015 \\
\hline & & Median & 11.72 & 5.80 & 2.02 & 0.043 \\
\hline \multirow{23}{*}{$\begin{array}{l}\text { ID Song vs. ID } \\
\text { Speech }\end{array}$} & Attack Curve Slope & Median & -0.81 & 0.25 & -3.29 & 0.001 \\
\hline & First Formant & Median & -33.81 & 6.47 & -5.23 & $<.001$ \\
\hline & Inharmonicity & Whole & -0.01 & 0.00 & -2.02 & 0.043 \\
\hline & Intensity Rate & Whole & -3.92 & 0.36 & -10.89 & $<.001$ \\
\hline & & IQR & -5.03 & 0.43 & -11.61 & $<.001$ \\
\hline & & Median & -2.11 & 0.17 & -12.25 & $<.001$ \\
\hline & Intensity Space & IQR & -1.33 & 0.16 & -8.46 & $<.001$ \\
\hline & & Median & -0.83 & 0.07 & -11.32 & $<.001$ \\
\hline & nPVI per Phrase & Whole & 4.39 & 1.14 & 3.87 & $<.001$ \\
\hline & nPVI per & Whole & 4.81 & 0.88 & 5.45 & $<.001$ \\
\hline & Recording & & & & & \\
\hline & Pitch Rate & Whole & -28.11 & 2.54 & -11.05 & $<.001$ \\
\hline & & IQR & -31.97 & 2.56 & -12.51 & $<.001$ \\
\hline & & Median & -5.78 & 0.56 & -10.25 & $<.001$ \\
\hline & Pitch Space & IQR & -9.99 & 0.77 & -12.94 & $<.001$ \\
\hline & & Median & -2.70 & 0.23 & -11.63 & $<.001$ \\
\hline & Roughness & IQR & -6.63 & 2.04 & -3.25 & 0.001 \\
\hline & & Median & -1.52 & 0.73 & -2.07 & 0.038 \\
\hline & Vowel Space & IQR & -55.11 & 7.82 & -7.05 & $<.001$ \\
\hline & & Median & -31.27 & 3.98 & -7.87 & $<.001$ \\
\hline & Vowel Space Travel & Whole & -227.44 & 21.26 & -10.70 & $<.001$ \\
\hline & Rate & IQR & -310.78 & 27.95 & -11.12 & $<.001$ \\
\hline & & Median & -124.53 & 11.05 & -11.27 & $<.001$ \\
\hline
\end{tabular}

Table S4. Significant results from confirmatory analyses, after Winsorization and excluding variables with extreme observations (e.g., using median and IQR instead of mean and standard deviation), using post-hoc linear combinations following multi-level mixed-effects models. 


\begin{tabular}{lrrr}
\hline Feature & $F(1,1094)$ & $p$ & $R^{2}$ \\
\hline Pitch (Median) & 489.9 & $5.27 \times 10^{-90}$ & 0.309 \\
Pitch (IQR) & 411.6 & $6.30 \times 10^{-78}$ & 0.273 \\
Intensity Space (Median) & 149.5 & $2.61 \times 10^{-32}$ & 0.120 \\
Temporal Modulation & 74.5 & $2.16 \times 10^{-17}$ & 0.064 \\
Roughness (IQR) & 69.9 & $1.86 \times 10^{-16}$ & 0.060 \\
Inharmonicity & 51.1 & $1.59 \times 10^{-12}$ & 0.045 \\
Roughness (Median) & 49.2 & $4.05 \times 10^{-12}$ & 0.043 \\
Pitch Space (IQR) & 29.2 & $7.89 \times 10^{-8}$ & 0.026 \\
Attack Curve Slope (Median) & 29.0 & $8.74 \times 10^{-8}$ & 0.026 \\
Energy Roll-off (85th \%ile) & 26.6 & $2.92 \times 10^{-7}$ & 0.024 \\
First Formant (Median) & 19.4 & $1.14 \times 10^{-5}$ & 0.017 \\
nPVI (per phrase) & 13.1 & $3.01 \times 10^{-4}$ & 0.012 \\
\hline
\end{tabular}

\section{Table S5.}

Omnibus tests from simple linear regressions of perceived infant-directedness (from the naive listener experiment) on each of 12 acoustic features validated by exploratoryconfirmatory and LASSO analyses. All tests are significant at the Bonferroni-corrected alpha level of .0024. 


\section{References}

1. Morton, E. S. On the occurrence and significance of motivation-structural rules in some bird and mammal sounds. The American Naturalist 111, 855-869 (1977).

2. Endler, J. A. Some general comments on the evolution and design of animal communication systems. Philosophical Transactions of the Royal Society B: Biological Sciences 340, 215-225 (1993).

3. Owren, M. J. \& Rendall, D. Sound on the rebound: Bringing form and function back to the forefront in understanding nonhuman primate vocal signaling. Evolutionary Anthropology 10, 58-71 (2001).

4. Fitch, W. T., Neubauer, J. \& Herzel, H. Calls out of chaos: The adaptive significance of nonlinear phenomena in mammalian vocal production. Animal Behaviour 63, 407-418 (2002).

5. Wiley, R. H. The evolution of communication: Information and manipulation. Animal Behaviour 2 , $156-189$ (1983).

6. Krebs, J. \& Dawkins, R. Animal signals: Mind-reading and manipulation. in Behavioural Ecology: An Evolutionary Approach (eds. Krebs, J. \& Davies, N.) 380-402 (Blackwell, 1984).

7. Karp, D., Manser, M. B., Wiley, E. M. \& Townsend, S. W. Nonlinearities in meerkat alarm calls prevent receivers from habituating. Ethology 120, 189-196 (2014).

8. Slaughter, E. I., Berlin, E. R., Bower, J. T. \& Blumstein, D. T. A test of the nonlinearity hypothesis in great-tailed grackles (Quiscalus mexicanus). Ethology 119, 309-315 (2013).

9. Wagner, W. E. Fighting, assessment, and frequency alteration in Blanchard's cricket frog. Behavioral Ecology and Sociobiology 25, 429-436 (1989).

10. Ladich, F. Sound production by the river bullhead, Cottus gobio L. (Cottidae, Teleostei). Journal of Fish Biology 35, 531-538 (1989).

11. Filippi, P. et al. Humans recognize emotional arousal in vocalizations across all classes of terrestrial vertebrates: Evidence for acoustic universals. Proceedings of the Royal Society B: Biological Sciences 284, (2017).

12. Lingle, S. \& Riede, T. Deer mothers are sensitive to infant distress vocalizations of diverse mammalian species. The American Naturalist 184, 510-522 (2014).

13. Custance, D. \& Mayer, J. Empathic-like responding by domestic dogs (Canis familiaris) to distress in humans: An exploratory study. Animal Cognition 15, 851-859 (2012).

14. Magrath, R. D., Haff, T. M., McLachlan, J. R. \& Igic, B. Wild birds learn to eavesdrop on heterospecific alarm calls. Current Biology 25, 2047-2050 (2015).

15. Lea, A. J., Barrera, J. P., Tom, L. M. \& Blumstein, D. T. Heterospecific eavesdropping in a nonsocial species. Behavioral Ecology 19, 1041-1046 (2008).

16. Soltis, J. The signal functions of early infant crying. Behavioral and Brain Sciences 27, 443-458 (2004).

17. Bryant, G. A. \& Barrett, H. C. Recognizing intentions in infant-directed speech: Evidence for universals. Psychological Science 18, 746-751 (2007).

18. Piazza, E. A., Iordan, M. C. \& Lew-Williams, C. Mothers consistently alter their unique vocal fingerprints when communicating with infants. Current Biology 27, 3162-3167 (2017).

19. Trehub, S. E., Unyk, A. M. \& Trainor, L. J. Adults identify infant-directed music across cultures. Infant Behavior and Development 16, 193-211 (1993).

20. Mehr, S. A., Singh, M., York, H., Glowacki, L. \& Krasnow, M. M. Form and function in human song. Current Biology 28, 356-368 (2018).

21. Mehr, S. A. et al. Universality and diversity in human song. Science 366, 957-970 (2019). 
22. Fernald, A. Human maternal vocalizations to infants as biologically relevant signals: An evolutionary perspective. in The adapted mind: Evolutionary psychology and the generation of culture (eds. Barkow, J. H., Cosmides, L. \& Tooby, J.) 391-428 (Oxford University Press, 1992).

23. Burnham, E., Gamache, J. L., Bergeson, T. \& Dilley, L. Voice-onset time in infant-directed speech over the first year and a half. in Proceedings of Meetings on Acoustics ICA2013 19, 060094 (ASA, 2013).

24. Fernald, A. Prosody in speech to children: Prelinguistic and linguistic functions. Annals of Child Development 8, 43-80 (1991).

25. Ferguson, C. A. Baby talk in six languages. American Anthropologist 66, 103-114 (1964).

26. Audibert, N. \& Falk, S. Vowel space and f0 characteristics of infant-directed singing and speech. in Proceedings of the 19th international conference on speech prosody 153-157 (2018).

27. Ratner, N. B. Phonological rule usage in mother-child speech. Journal of Phonetics 12, 245-254 (1984).

28. Kuhl, P. K. et al. Cross-language analysis of phonetic units in language addressed to infants. Science 277, 684-686 (1997).

29. Englund, K. T. \& Behne, D. M. Infant directed speech in natural interaction: Norwegian vowel quantity and quality. Journal of Psycholinguistic Research 34, 259-280 (2005).

30. Fernald, A. \& Simon, T. Expanded intonation contours in mothers' speech to newborns. Developmental Psychology 20, 104-113 (1984).

31. Falk, S. \& Kello, C. T. Hierarchical organization in the temporal structure of infant-direct speech and song. Cognition 163, 80-86 (2017).

32. Thiessen, E. D., Hill, E. A. \& Saffran, J. R. Infant-directed speech facilitates word segmentation. Infancy 7, 53-71 (2005).

33. Trainor, L. J. \& Desjardins, R. N. Pitch characteristics of infant-directed speech affect infants' ability to discriminate vowels. Psychonomic Bulletin \& Review 9, 335-340 (2002).

34. Werker, J. F. \& McLeod, P. J. Infant preference for both male and female infant-directed talk: A developmental study of attentional and affective responsiveness. Canadian Journal of Psychology/Revue Canadienne de Psychologie 43, 230-246 (1989).

35. Falk, D. Prelinguistic evolution in early hominins: Whence motherese? Behavioral and Brain Sciences 27, 491-502 (2004).

36. ManyBabies Consortium. Quantifying sources of variability in infancy research using the infant-directedspeech preference. Advances in Methods and Practices in Psychological Science 3, 24-52 (2020).

37. Soley, G. \& Sebastian-Galles, N. Infants' expectations about the recipients of infant-directed and adultdirected speech. Cognition 198, 104214 (2020).

38. Henrich, J., Heine, S. J. \& Norenzayan, A. The weirdest people in the world? Behavioral and Brain Sciences 33, 61-83 (2010).

39. Bowlby, J. Attachment and Loss (Vol. I: Attachment). (Basic Books, 1969).

40. Konner, M. The evolution of childhood: Relationships, emotion, mind. (Belknap Press of Harvard University Press, 2010).

41. Grieser, D. L. \& Kuhl, P. K. Maternal speech to infants in a tonal language: Support for universal prosodic features in motherese. Developmental Psychology 24, 14 (1988).

42. Fisher, C. \& Tokura, H. Acoustic cues to grammatical structure in infant-directed speech: Cross-linguistic evidence. Child Development 67, 3192-3218 (1996).

43. Fernald, A. et al. A cross-language study of prosodic modifications in mothers' and fathers' speech to preverbal infants. Journal of Child Language 16, 477-501 (1989). 
44. Broesch, T. L. \& Bryant, G. A. Prosody in infant-directed speech is similar across western and traditional cultures. Journal of Cognition and Development 16, 31-43 (2015).

45. Farran, L. K., Lee, C.-C., Yoo, H. \& Oller, D. K. Cross-cultural register differences in infant-directed speech: An initial study. PLoS ONE 11, (2016).

46. Broesch, T. \& Bryant, G. A. Fathers' infant-directed speech in a small-scale society. Child Development 89, e29-e41 (2018).

47. Cristia, A., Dupoux, E., Gurven, M. \& Stieglitz, J. Child-directed speech is infrequent in a forager-farmer population: A time allocation study. Child Development 90, 759-773 (2019).

48. Konner, M. Infancy among the Kalahari desert San. in Culture and Infancy: Variations in the Human Experience (eds. Leiderman, H., Tulkin, S. R. \& Rosenfeld, A. H.) 287-328 (Academic Press, 1977).

49. Mehr, S. A. \& Krasnow, M. M. Parent-offspring conflict and the evolution of infant-directed song. Evolution and Human Behavior 38, 674-684 (2017).

50. Cirelli, L. K. \& Trehub, S. E. Familiar songs reduce infant distress. Developmental Psychology (2020). doi:10.1037/dev0000917

51. Bainbridge, C. et al. Infants relax in response to unfamiliar foreign lullabies. PsyArXiv (2020). doi:10.31234/osf.io/xcj52

52. Corbeil, M., Trehub, S. E. \& Peretz, I. Singing delays the onset of infant distress. Infancy 21, 373-391 (2016).

53. Cassidy, S. B. \& Driscoll, D. J. Prader-Willi syndrome. European Journal of Human Genetics 17, 3-13 (2008).

54. Williams, C. A. et al. Angelman syndrome 2005: Updated consensus for diagnostic criteria. American Journal of Medical Genetics 140, 413-418 (2006).

55. Mehr, S. A., Kotler, J., Howard, R. M., Haig, D. \& Krasnow, M. M. Genomic imprinting is implicated in the psychology of music. Psychological Science 28, 1455-1467 (2017).

56. Kotler, J., Mehr, S. A., Egner, A., Haig, D. \& Krasnow, M. M. Response to vocal music in Angelman syndrome contrasts with Prader-Willi syndrome. Evolution and Human Behavior 40, 420-426 (2019).

57. Trehub, S. E. Musical predispositions in infancy. Annals of the New York Academy of Sciences 930, $1-16$ (2001).

58. Peretz, I. The nature of music from a biological perspective. Cognition 100, 1-32 (2006).

59. McDermott, J. \& Hauser, M. The origins of music: Innateness, uniqueness, and evolution. Music Perception 23, 29-59 (2005).

60. Fan, S. et al. African evolutionary history inferred from whole genome sequence data of 44 indigenous African populations. Genome Biology 20, 82 (2019).

61. Konner, M. Aspects of the developmental ethology of a foraging people. in Ethological Studies of Child Behaviour (ed. Blurton Jones, N. G.) 285-304 (Cambridge University Press, 1972).

62. Marlowe, F. The Hadza hunter-gatherers of Tanzania. (University of California Press, 2010).

63. Trehub, S. E. \& Trainor, L. Singing to infants: Lullabies and play songs. Advances in Infancy Research 12, 43-78 (1998).

64. Trehub, S. E. et al. Mothers' and fathers' singing to infants. Developmental Psychology 33, 500-507 (1997).

65. Trehub, S. E., Hill, D. S. \& Kamenetsky, S. B. Parents' sung performances for infants. Canadian Journal of Experimental Psychology 51, 385-396 (1997).

66. Boersma, P. W. Praat: Doing phonetics by computer. (2019). 
67. de Leeuw, J. R. jsPsych: A JavaScript library for creating behavioral experiments in a Web browser. Behavior Research Methods 47, 1-12 (2015).

68. Hartshorne, J. K., de Leeuw, J., Goodman, N., Jennings, M. \& O’Donnell, T. J. A thousand studies for the price of one: Accelerating psychological science with Pushkin. Behavior Research Methods 51, 1782-1803 (2019).

69. Lartillot, O., Toiviainen, P. \& Eerola, T. A Matlab toolbox for music information retrieval. in Data analysis, machine learning and applications (eds. Preisach, C., Burkhardt, H., Schmidt-Thieme, L. \& Decker, R.) 261-268 (Springer Berlin Heidelberg, 2008).

70. Patel, A. D., Iversen, J. R. \& Rosenberg, J. C. Comparing the rhythm and melody of speech and music: The case of British English and French. The Journal of the Acoustical Society of America 119, 3034 (2006).

71. Mertens, P. The prosogram: Semi-automatic transcription of prosody based on a tonal perception model. in Speech Prosody 2004, International Conference (2004).

72. Friedman, J., Hastie, T. \& Tibshirani, R. Lasso and elastic-net regularized generalized linear models. Rpackage version 2.0-5. (2016).

73. Yale, C. \& Forsythe, A. B. Winsorized regression. Technometrics 18, 291-300 (1976).

74. Salselas, I. \& Herrera, P. Development of perception and representation of rhythmic information: Towards a computational model. in 2010 9th International Conference on Development and Learning (IEEE, 2010).

75. Arnal, L. H., Flinker, A., Kleinschmidt, A., Giraud, A.-L. \& Poeppel, D. Human screams occupy a privileged niche in the communication soundscape. Current Biology 25, 2051-2056 (2015).

76. Diehl, R. L. Acoustic and auditory phonetics: The adaptive design of speech sound systems. Philosophical Transactions of the Royal Society B: Biological Sciences 363, 965-978 (2007).

77. Nadeau, C. \& Bengio, Y. Inference for the generalization error. Machine Learning 52, 239-281 (2003).

78. Blumstein, D. T., Bryant, G. A. \& Kaye, P. The sound of arousal in music is context-dependent. Biology Letters 8, 744-747 (2012).

79. Polka, L. \& Werker, J. F. Developmental changes in perception of nonnative vowel contrasts. Journal of Experimental Psychology: Human Perception and Performance 20, 421-435 (1994).

80. Bertoncini, J., Bijeljac-Babic, R., Jusczyk, P. W., Kennedy, L. J. \& Mehler, J. An investigation of young infants' perceptual representations of speech sounds. Journal of Experimental Psychology: General 117, 21-33 (1988).

81. Werker, J. F. \& Lalonde, C. E. Cross-language speech perception: Initial capabilities and developmental change. Developmental Psychology 24, 672 (1988).

82. Buyens, W., Moonen, M., Wouters, J. \& van Dijk, B. A model for music complexity applied to music preprocessing for cochlear implants. in 2017 25th European Signal Processing Conference (EUSIPCO) 971975 (IEEE, 2017).

83. Ding, N. et al. Temporal modulations in speech and music. Neuroscience 83 Biobehavioral Reviews 81, (2017). 